\title{
MHD convection in a partially driven cavity with corner heating
}

\author{
Milan K. Mondal ${ }^{1}$ - Nirmalendu Biswas ${ }^{2} \cdot$ Nirmal K. Manna $^{3}$
}

Received: 13 May 2019 / Accepted: 16 November 2019 / Published online: 26 November 2019

(c) Springer Nature Switzerland AG 2019

\begin{abstract}
The paper presents a pertinent modification to the wall-moving thermal systems (classically represented by lid-driven cavity) by introducing a partial motion of the boundary walls. The effect of partial wall motion is investigated under magnetohydrodynamic (MHD) mixed convection in the cavity filled with porous medium. The corner heating arrangement is chosen for this investigation. The heater is placed at the right-bottom corner of the cavity, whereas the halves of the adjacent sides (which are moving) of the top-left corner are utilized for cooling. The present MHD thermofluid flow in porous cavity under partially driven condition is solved numerically using Brinkman-Forchheimer-Darcy model and analyzed using heatlines, streamlines, isotherms and average Nusselt number. The detailed insights of partially driven cavity are captured for different values of Richardson number ( $\mathrm{R} i=0.1-100)$, Reynolds number ( $\mathrm{Re}=10-500)$, Hartmann number $(\mathrm{Ha}=0-100)$, Darcy number $\left(\mathrm{Da}=10^{-7}\right.$ to $\left.10^{-3}\right)$ and porosity $(\varepsilon=0.1-1.0)$. The result reveals that the partial wall motion and its direction have a significant impact on the thermofluid flow structures and heat transfer rate.
\end{abstract}

\section{Graphic abstract}

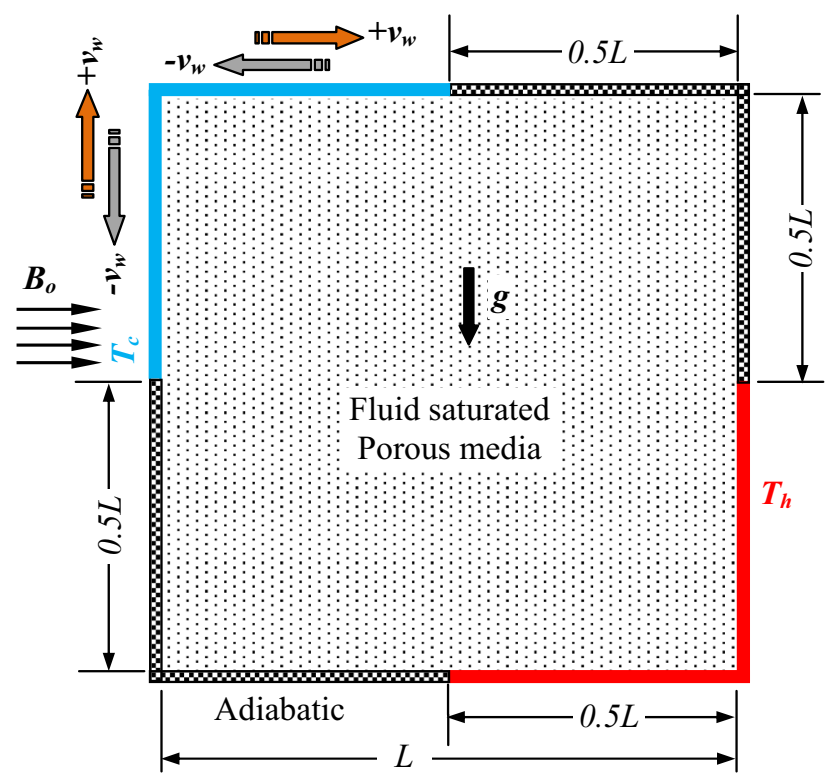

$\triangle$ Nirmal K. Manna, nirmalkmannaju@gmail.com; Milan K. Mondal, milanmondal@gmail.com; Nirmalendu Biswas, nirmalendubiswas@ yahoo.co.in | 1 Department of Mechanical Engineering, MSG Polytechnic, Paschim Medinipur 721102, India. ${ }^{2}$ Department of Power Engineering, Jadavpur University, Salt Lake, Kolkata 700098, India. ${ }^{3}$ Department of Mechanical Engineering, Jadavpur University, Kolkata 700032, India. 
Keywords Magnetohydrodynamic (MHD) convection · Partially driven cavity (PDC) · Porous cavity · Corner heating · Heatlines

\section{List of symbols}

$B_{\mathrm{o}} \quad$ Magnitude of magnetic field

Da Darcy number

$F_{C} \quad$ Forchheimer coefficient

$g$ Acceleration due to gravity $\left(\mathrm{m} \mathrm{s}^{-2}\right)$

Gr Grashof number

Ha Hartmann number

$K \quad$ Permeability of porous medium $\left(\mathrm{m}^{2}\right)$

$L \quad$ Length scale $(\mathrm{m})$

$\mathrm{Nu}$ Average Nusselt number

$P \quad$ Dimensionless pressure

Pr Prandtl number

Re Reynolds number

$\mathrm{Ri}$ Richardson number

$T$ Temperature (K)

$u, v \quad$ Velocity components $\left(\mathrm{m} \mathrm{s}^{-1}\right)$

$U, V$ Dimensionless velocity components

$v_{w} \quad$ Dimensionless translational speed

$V_{w} \quad$ Translational speed $\left(\mathrm{m} \mathrm{s}^{-1}\right)$

$x, y \quad$ Cartesian coordinates $(\mathrm{m})$

$X, Y$ Dimensionless coordinates

\section{Greek symbols}

$\beta \quad$ Thermal expansion coefficient $\left(\mathrm{K}^{-1}\right)$

$\theta \quad$ Dimensionless temperature

$\varepsilon \quad$ Porosity

$v \quad$ Kinematic viscosity $\left(\mathrm{m}^{2} \mathrm{~s}^{-1}\right)$

$\Pi$ Dimensionless heat function

$\rho \quad$ Density $\left(\mathrm{kg} \mathrm{m}^{-3}\right)$

$\kappa \quad$ Electrical conductivity $\left(\mu \mathrm{S} \mathrm{cm}^{-1}\right)$

$\psi \quad$ Dimensionless stream function

\section{Subscripts}

c Cold wall

$h$ Hot wall

\section{Introduction}

The combination of forced and natural convection, commonly termed as mixed convection, is a useful technique for heat transfer in cavity-like thermal systems. The forced convective flow may originate from either external flow or boundary wall motion. In a typical lid-driven or moving wall cavity, the mixed convective flow originates from two mechanisms-shearing action due to wall motion (sheared or forced flow) and buoyancy-induced flow due to the presence of thermal gradients (free or natural convection). In addition, the convective flow dynamics is greatly suffered in the presence of magnetic fields. The combined situation (mixed convection and magnetic fields) may be called magnetohydrodynamic (MHD) convection. By adjusting the strength of the magnetic field, the thermohydrodynamics as well as heat transfer characteristics can be controlled as per the requirement of a specific process. The systems involving MHD convection are encountered in a variety of technological processessuch as material processing, crystal growth, solidification, nuclear reactors, air-conditioning, microelectronic devices, food processing, coating, etc. $[1,2]$. A comprehensive review about the application of magnetic fields in biomedical science has been reported in Rashidi et al. [3].

Many investigations on mixed convection caused by the wall motion of porous cavity along with the magnetic field, have been reported in the literature. Oztop et al. [4] numerically analyzed MHD mixed convection due to the top wall motion of an enclosure heated at the corner. Later on, Ray et al. [5] conducted numerical analysis of the above problem considering a round body placed at the center (of the cavity) and Joule heating. They reported that the variation of magnetic field can effectively control the flow characteristics. The driven cavity heated at the corner has been studied by Abu-Nada [6]. Very recently, Ismael et al. [7] numerically studied a similar configuration of lid-driven cavity using CuO-water nanofluid. The effect of corner heating and lid motion on MHD mixed convection in cavity has also been studied by Malleswaran and Sivasankaran [8]. They reported about the effect of heater length on heat transfer as well as on the flow structure. Begum et al. [9] made a computation of MHD mixed convection of nanofluid (Cu-water) saturated porous medium in the presence of an inclined uniform magnetic field in a rectangular cavity (which is heated sinusoidally at sidewalls and has an isothermally heated moving lid at center). They found that the effect of magnetic field as well as its orientation had adverse impact on the overall heat transfer rate. The consideration of corner heating for closed cavities has been reported in a number of works [10-13]. Other notable works in MHD convective flow can be found in Hussain et al. [14] and Gibanov et al. [15]. Very recently, Chamkha et al. [16] studied MHD convective heat transfer of $\mathrm{Cu}$-water nanofluid saturated porous medium considering heat generation/absorption. Convective heat transfer in cavities has also been studied by Bondarenko et al. [17] considering two adherent porous blocks in a lid-driven cavity and Maghsoudi and Siavashi [18] considering a twosided lid-driven porous cavity. Akar et al. [19] have analyzed turbulent fluid flow around a rotating cylinder and evaluated entropy generation. In other classes of works on convection lid-driven cavities have considered porous media with no-magnetic field [20], magnetic fields in the 
absence of porous substance [20-22] and magnetic fields along with porous substrate $[16,23,24]$.

The applications and importance of corner-heateddriven cavity or corner-heated cavity along with magnetic fields and porous medium, are clearly indicated in the above-mentioned literature. Magnetohydrodynamic convection finds countless applications with and without porous medium. Many engineering problems are represented using enclosures/cavities having all walls stationary or one or more walls moving. Customarily, a whole wall of a driven cavity is utilized for the translational motion. However, there are many situations where providing motion to the entire wall may not possible (for the providing support or some other reasons). Here, we propose a novel modification of lid-driven cavity with partial wall motion by dividing a whole wall into two parts: one part stationary and other part moving. So, we refer it as a partially driven cavity in this work along with the consideration of other important physical aspects (like porous medium, magnetic fields) covering a wide area of practical applications. In between two established cavity-like thermal systems, the stationary cavity and the driven cavity, the idea of a partially driven cavity (in brief, PDC) is in between and could meaningfully be utilized where the motion of a whole wall is restricted (or not feasible from the application point of view). It could open up a new area of research. The present study is carried out from the fundamental point of view, to explore the impacts of partial wall motion on the magnetothermohydrodynamics in cavity in parametric form using Reynolds number, Richardson number, Hartmann number, Darcy number and porosity. The heatlines, streamlines, isotherms and the average Nusselt number are utilized in the investigation.

\section{Modeling aspects}

Figure 1 shows the chosen problem, a partially driven square cavity (of height ' $L$ ') with corner heating (at the right-bottom corner) and corner cooling (at the upperleft corner). The halves of the adjacent sides $(0.5 \mathrm{~L}$ each) at the right-bottom corner are used as heater assuming a constant temperature $\left(T_{\mathrm{h}}\right)$ of heating. On the other hand, the halves of the adjacent sides $(0.5 \mathrm{~L}$ each) at the upperleft corner are used as cooler assuming a constant temperature $\left(T_{\mathrm{c}}\right)$ of cooling. These two halves of the cooler can translate, keeping the remaining halves of the boundary wall stationary. The impacts of the direction of moving (left/right and downward/upward) and the speed $\left(V_{w}\right)$ are also taken into account in this work. Apart from the heating and cooling portions, the rest of the walls of the cavity are adiabatic. The cavity contains porous medium saturated with an electrically conducting fluid of $\operatorname{Pr}=0.71$.

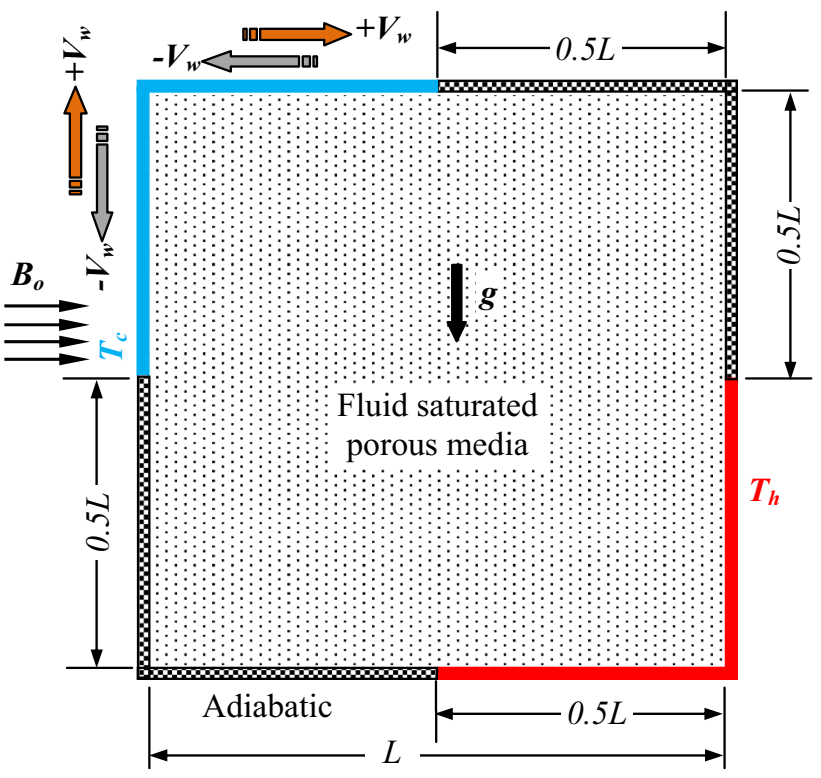

Fig. 1 (Color online) Schematic diagram of the physical domain with boundary conditions

The uniform magnetic fields $\left(B_{\mathrm{o}}\right)$ are applied on the cavity in the horizontal direction from an external magnetic source. However, the porous medium and the solid walls are assumed to be electrically non-conducting [4]. The induced magnetic field from the electrically conducting fluid and the Hall effect are neglected [21, 22, 24]. The induced magnetic field is ignored due to the small value of magnetic Reynolds number, which generally results from partially ionized gaseous substance used as a working fluid. Secondly, the Hall effect generally comes into play when the magnetic field is very strong. The porous substance is assumed to be isotropic and homogeneous along with uniform porosity $(\varepsilon)$. The momentum equations are formulated taking care of the inertial frictional effect using Brinkman-Forchheimer-Darcy model (BFDM). Furthermore, the local thermal equilibrium (LTE) model is utilized to formulate the energy equation neglecting insignificant viscous dissipation $[4,5,25]$. The present study is conducted for a steady Newtonian laminar flow of an incompressible fluid. The buoyancy force is modeled following the Boussinesq approximation.

Based on the above-mentioned facts, the governing equations are derived for a two-dimensional flow in the cavity. For the parametric study, these equations (for continuity, momentum and energy) are non-dimensionalized using a length scale $L$, a velocity scale $V_{w^{\prime}}$ a dimensionless pressure $P\left[=\left(p-p_{a}\right) / \rho V_{w}^{2}\right]$ and a dimensionless temperature $\theta\left[=\left(T-T_{c}\right) /\left(T_{h}-T_{c}\right)\right]$. The resulting dimensionless equations are 
$\frac{\partial U}{\partial X}+\frac{\partial V}{\partial Y}=0$

$\frac{1}{\varepsilon^{2}}\left(U \frac{\partial U}{\partial X}+V \frac{\partial U}{\partial Y}\right)=-\frac{\partial P}{\partial X}+\frac{1}{\operatorname{Re} \varepsilon}\left(\frac{\partial^{2} U}{\partial X^{2}}+\frac{\partial^{2} U}{\partial Y^{2}}\right)$

$$
-\left(\frac{1}{\mathrm{DaRe}}+\frac{F_{C} \sqrt{U^{2}+V^{2}}}{\sqrt{\mathrm{Da}} \varepsilon^{3 / 2}}\right) U
$$

$\frac{1}{\varepsilon^{2}}\left(U \frac{\partial V}{\partial X}+V \frac{\partial V}{\partial Y}\right)=-\frac{\partial P}{\partial Y}+\frac{1}{\operatorname{Re} \varepsilon}\left(\frac{\partial^{2} V}{\partial X^{2}}+\frac{\partial^{2} V}{\partial Y^{2}}\right)$

$$
-\left(\frac{1}{\mathrm{DaRe}}+\frac{F_{C} \sqrt{U^{2}+V^{2}}}{\sqrt{\mathrm{Da}} \varepsilon^{3 / 2}}\right) V
$$$$
-\frac{\mathrm{Ha}{ }^{2}}{\operatorname{Re}} V+\operatorname{Ri} \theta
$$

$\left(U \frac{\partial \theta}{\partial X}+V \frac{\partial \theta}{\partial Y}\right)=\frac{1}{\operatorname{Re} \operatorname{Pr}}\left(\frac{\partial^{2} \theta}{\partial X^{2}}+\frac{\partial^{2} \theta}{\partial Y^{2}}\right)$

where $\mathrm{Re}, \mathrm{Da}, \mathrm{Ha}, \mathrm{Ri}, \operatorname{Pr}$ and $F_{\mathrm{C}}(=1.75 / \sqrt{150})$ are, respectively, Reynolds, Darcy, Hartmann, Richardson and Prandtl numbers, and Forchheimer coefficient. These numbers are defined by

$\mathrm{Re}=\frac{V_{\mathrm{w}} L}{v}, \quad \mathrm{Da}=\frac{K}{L^{2}}, \quad \mathrm{Ha}=\frac{B_{\mathrm{o}} L}{\sqrt{\rho v / \kappa}}, \quad \mathrm{Ri}=\frac{\mathrm{Gr}}{\mathrm{Re}^{2}}, \quad \operatorname{Pr}=\frac{v}{\alpha}$

where Grashof number $\mathrm{Gr}=g \beta\left(T_{\mathrm{h}}-T_{\mathrm{c}}\right) L^{3} / \nu^{2}$.

Governing Eqs. (1)-(4) are subjected to the following boundary conditions:

1. at the left wall, for the lower half $(X=0$ and $0 \leq Y<0.5), U=V=0, \partial \theta / \partial X=0$ and for the upper half $(X=0$ and $0.5 \leq Y \leq 1), U=0, V= \pm 1, \theta=0$

2. at the right wall, for the lower half ( $X=1$ and $0 \leq Y<0.5), U=V=0, \theta=1$ and for the upper half $(X=0$ and $0.5 \leq Y \leq 1), U=V=0, \partial \theta / \partial X=0$

3. at the bottom wall, for the first half $(Y=0,0 \leq X<0.5)$, $U=V=0, \partial \theta / \partial Y=0$ and for the last half $(Y=0$, $0.5 \leq X \leq 1), U=V=0, \theta=1$

4. at the top wall, for the first half $(Y=1,0 \leq X<0.5)$, $U= \pm 1, V=0, \theta=0$ and for the last half $(Y=1$, $0.5 \leq X \leq 1), U=V=0, \partial \theta / \partial Y=0$

Under the steady state, the rate of heat transfer from both the heater and the cooler is the same. It is computed and presented here in a non-dimensional form using the average Nusselt number $(\mathrm{Nu})$ for the cooling parts of the walls as
$\mathrm{Nu}=\int_{0}^{0.5}\left(-\left.\frac{\partial \theta}{\partial Y}\right|_{Y=1}\right) \mathrm{d} X+\int_{0.5}^{1}\left(\left.\frac{\partial \theta}{\partial X}\right|_{X=0}\right) \mathrm{d} Y$

The understanding of heat transport mechanism from the heat source to the heat sink is another important issue, for which the Bejan's heatlines [26] are utilized in the analysis. The heatlines are generated from the heatfunction ( $\Pi)$ which takes care of both convective and conductive heat fluxes under the steady-state condition of energy equation. For the present MHD mixed convective flow, the gradients of heatfunction are expressed by

$-\frac{\partial \Pi}{\partial X}=V \theta-\frac{1}{\operatorname{Re} \operatorname{Pr}} \frac{\partial \theta}{\partial Y}$ and $\frac{\partial \Pi}{\partial Y}=U \theta-\frac{1}{\operatorname{Re} \operatorname{Pr}} \frac{\partial \theta}{\partial X}$

For the solution of Eq. (7), the reference value of $\Pi$ is taken zero at the lower-left corner $(X=0, Y=0)$ of the cavity. The detailed procedure of solution is discussed in Biswas et al. [27]. Furthermore, the fluid-flow structure in the cavity is explored by the streamfunction $\psi$ which is used to generate streamlines.

\section{Numerical procedure}

The dimensionless partial differential Eqs. (1)-(4) are solved numerically using appropriate initial and boundary conditions through a developed CFD code. The finite volume method is adopted to discretize the governing equations which are solved iteratively using SIMPLE algorithm [28] and tridiagonal matrix algorithm (TDMA). The final solutions are obtained when the maximum values of continuity mass defect and residuals (of different equations) become below $10^{-10}$ and $10^{-8}$, respectively. This code has already been validated extensively during our earlier works $[27,29]$ on natural convection as well as mixed convection. Furthermore, a case of MHD thermal convection in a cavity for $\mathrm{Ra}=10^{7}, \mathrm{Ha}=30, \mathrm{Pr}=6.2$ as reported in Ghasemi et al. [30] has been validated. The published results and the simulated results are presented in Table 1 using the average Nusselt number $\mathrm{(Nu}$ ) and the maximum streamfunction $\left(|\psi|_{\max }\right)$. The table shows a good agreement in between these two results.

The test of grid independence is conducted for the present problem of partially driven and corner-heated cavity using non-uniform grids. Considering different values of $\mathrm{Ri}$ and fixed values of $\mathrm{Re}=200, \mathrm{Da}=10^{-4}, \varepsilon=0.6$, $\mathrm{Ha}=30$ and $v_{\mathrm{w}}=+1$, the problem is solved on different grid systems (coarse to fine grids) of the sizes of $90 \times 90$, $120 \times 120,150 \times 150$ and $200 \times 200$. These results are indicated in Table 2 using the average Nusselt number $(\mathrm{Nu})$ of the cooler. The successive changes in Nu value due to grid refinement are also shown in the brackets (which is 
Table 1 Average Nusselt number $(\mathrm{Nu})$ and maximum streamfunction $\left(|\psi|_{\text {max }}\right)$ of the published results [30] and the present simulated results at different $\mathrm{Ra}, \mathrm{Pr}=6.2$ and $\mathrm{Ha}=30$

\begin{tabular}{lccccc}
\hline Ra & \multicolumn{2}{l}{ Ghasemi et al. [30] } & & \multicolumn{2}{l}{ Present code } \\
\cline { 2 - 3 } & Nu & $|\psi|_{\max }$ & & Nu & $|\psi|_{\max }$ \\
\hline $10^{3}$ & 1.002 & 0.128 & & 1.003 & 0.128 \\
$10^{4}$ & 1.183 & 1.193 & & 1.187 & 1.192 \\
$10^{5}$ & 3.150 & 5.71 & & 3.163 & 5.755 \\
$10^{6}$ & 7.907 & 14.088 & & 7.986 & 14.297 \\
$10^{7}$ & 16.929 & 30.813 & & 17.281 & 31.287 \\
\hline
\end{tabular}

Table 2 Average Nusselt number (Nu) at $\mathrm{Re}=200, \mathrm{Ha}=30$, $\mathrm{Da}=10^{-4}, \varepsilon=0.6$ and $v_{\mathrm{w}}=+1$

\begin{tabular}{lllll}
\hline Ri & $90 \times 90$ & $120 \times 120$ & $150 \times 150$ & $200 \times 200$ \\
\hline 0.1 & 1.069 & $1.078(0.80 \%)$ & $1.069(0.86 \%)$ & $1.068(0.03 \%)$ \\
1 & 1.055 & $1.063(0.80 \%)$ & $1.054(0.84 \%)$ & $1.054(0.02 \%)$ \\
10 & 1.149 & $1.157(0.71 \%)$ & $1.147(0.93 \%)$ & $1.146(0.08 \%)$ \\
100 & 4.362 & $4.402(0.92 \%)$ & $4.386(0.36 \%)$ & $4.395(0.19 \%)$ \\
\hline
\end{tabular}

based on the preceding coarse grid). It is observed that for the grid sizes of $120 \times 120,150 \times 150$ and $200 \times 200$, the error is less than $1 \%$. So, all of them are suitable for the present computation. However, the grid size of $150 \times 150$ is selected for all the simulations to provide more grid points near the moving wall and to capture shear-driven corner vortex in a better way.

\section{Results and discussion}

The consideration of partial wall motion (by dividing a whole wall of a cavity into two parts: stationary and moving) along with different coupled physics is the main focus of this work. The problem geometry is briefly referred as PDC (partially driven cavity). It addresses multiphysical thermal systems involving porous substance, magnetic fields and buoyancy. The impacts of different physics of MHD mixed convection in PDC are investigated extensively within the limit of laminar flow and the Boussinesq approximation neglecting Hall effect. For this, the range of involved parameters is chosen as: Richardson number $(\mathrm{Ri}=0.1,1,10,100)$, Reynolds number $(\operatorname{Re}=10,100,200$, 500), Darcy number $\left(\mathrm{Da}=10^{-7}, 10^{-6}, 10^{-5}, 10^{-4}, 10^{-3}\right)$, porosity $(\varepsilon=0.1 .0 .3,0.5,0.6,0.8,1)$, Hartmann number $(\mathrm{Ha}=0,10,30,50,70,100)$. These ranges of parameters are found consistent with the available works [1-3] in the fields of lid-driven cavities, porous cavities and MHD convection in cavities. The direction of partial translation of the adjacent walls $\left(v_{w}= \pm 1\right)$ of the left-top corner is also included in the investigation. The results are presented systematically along with the explanations on fluid flow, temperature distribution, heat flow and heat transfer (using streamlines, isotherms, heatlines and average Nusselt number, respectively) in different subsections.

\subsection{Typical analysis of stationary and partially driven cavities}

To understand the advantage of partially driven cavity, two cavities in all respects are considered identical except one has moving half walls adjacent to the left-top corner only (PDC) as shown in Fig. 1 and other has all walls stationary. For the purpose of one-to-one comparison of transport phenomena in the cavities, the Grashof number $(\mathrm{Gr})$ is considered the same. The stationary cavity is simulated using the zero-velocity boundary condition for all the walls and $\operatorname{Re}=1 / \operatorname{Pr}$. It should be worth mentioning here that $R e$ in mixed convection governing equations given in Eqs. (1)-(4) when substituted by $1 / \mathrm{Pr}$, it directly transforms into natural convection governing equations. The impact of partial sliding motion of the adjacent walls on the heat and fluid-flow dynamics over the stationary cavity is demonstrated in Fig. 2 in terms of combined plot of streamlines and isotherms (blue lines-streamlines, dashed red lines-isotherms) and the plot of heatlines, given in the successive rows. The values of different parameters are: $\mathrm{Gr}=10^{4}, 10^{5}$ and $10^{6}, \mathrm{Da}=10^{-4}, \varepsilon=0.6$ and $\mathrm{Ha}=30$. The results for the stationary cavity and the partially driven cavity (PDC) are indicated in Fig. 2a, b, respectively.

It is observed from Fig. 2a that heating at the right-bottom corner causes buoyancy-driven flow upward along the right wall; after getting obstructed at the top wall, it passes over the cold left-top corner and finally descends along the left wall. Thus, a counter-clockwise (CCW) rotating circulation is formed in the cavity. Of course, as $\mathrm{Gr}$ value increases, the strength of the circulating vortex increases significantly - which is clearly reflected by the streamline contours at $\mathrm{Gr}=10^{4}, 10^{5}$ and $10^{6}$. Due to the heating and cooling at the diagonally opposite corners, the isotherms are inclined to the horizontal direction when $\mathrm{Gr} \leq 10^{5}$. When the circulation becomes stronger at $\mathrm{Gr}=10^{6}$, the isotherms are mostly oriented vertically. From the contours of heatlines (second row), it can be observed that heat energy from the heat source is transported to the cold corner walls directly when $\mathrm{Gr} \leq 10^{5}$ as indicated by heatlines. With the increase in $\mathrm{Gr}$ value to $10^{6}$, the energy circulation cell (rotating in counter-clockwise direction) develops inside the cavity. It is pertinent to mention here that the buoyancy flow is opposed by the magnetic force that dampens the vertical component of velocity directly. From the Nu values (as indicated below the heatlines contours), it is clear that the heat transfer substantially 

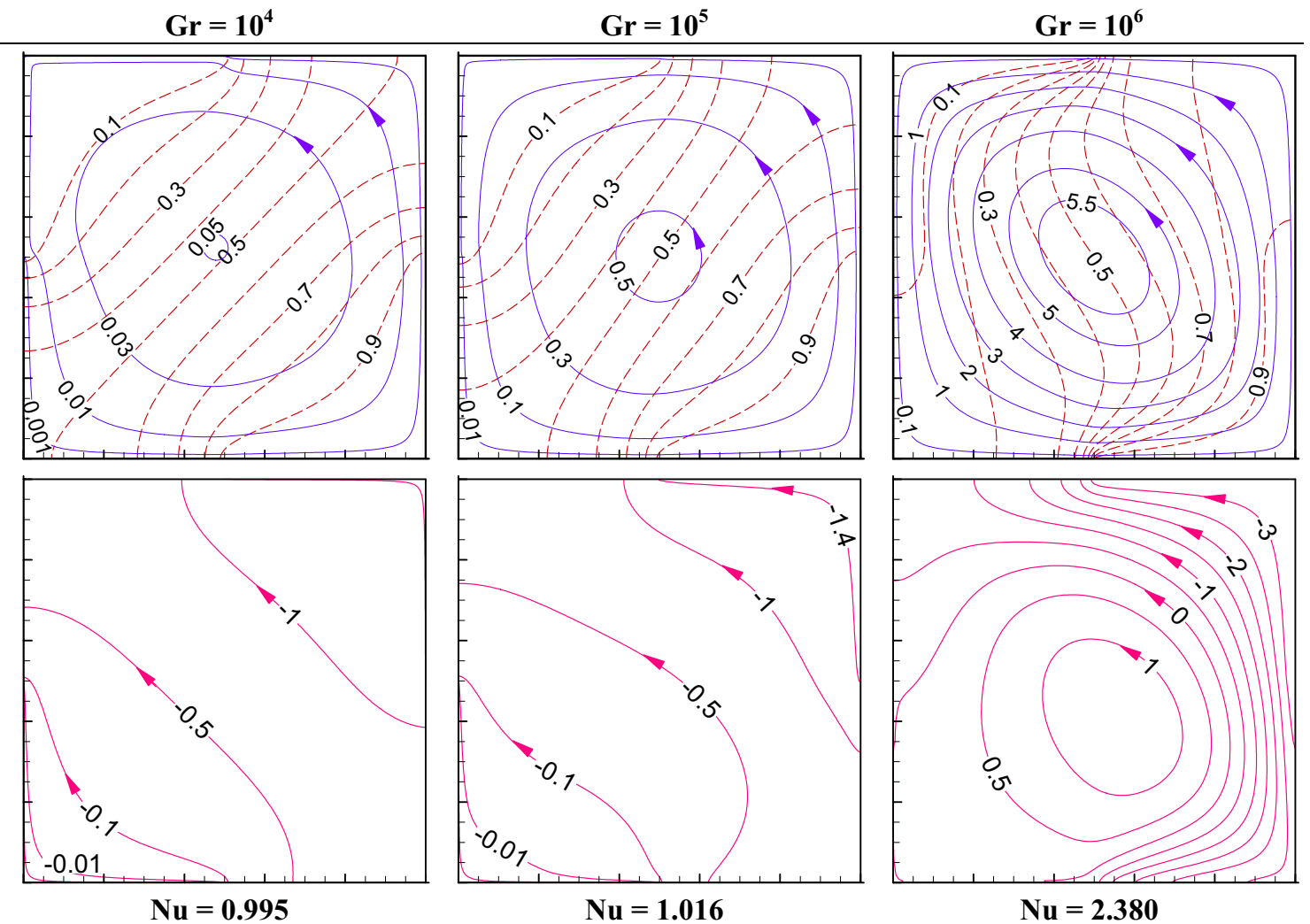

(a) Stationary cavity
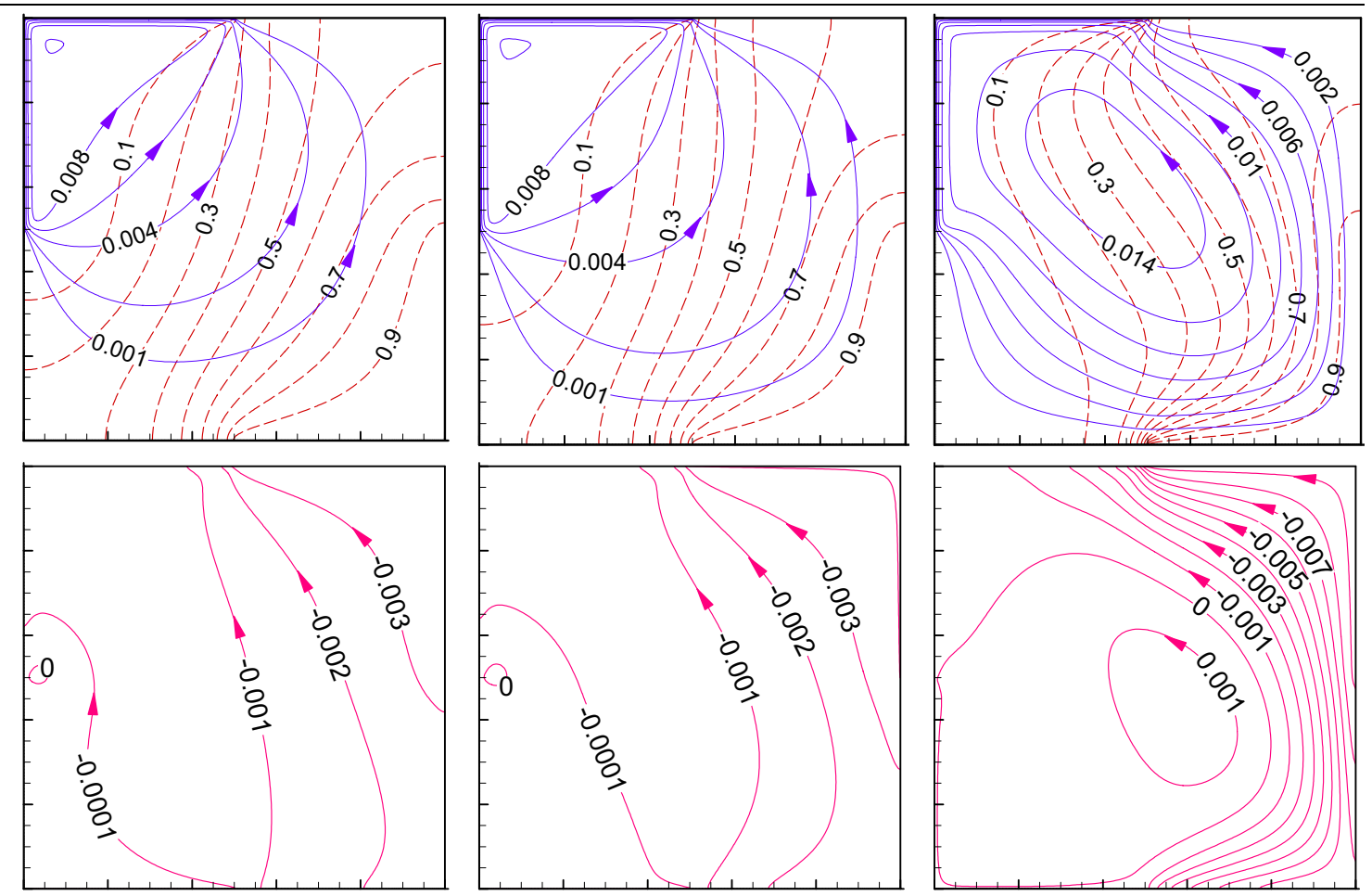

$\mathrm{Nu}=\mathbf{1 . 2 8 7}$

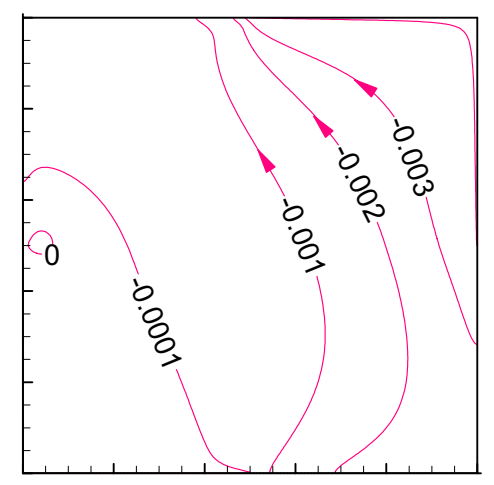

$\mathrm{Nu}=\mathbf{1 . 4 2 5}$

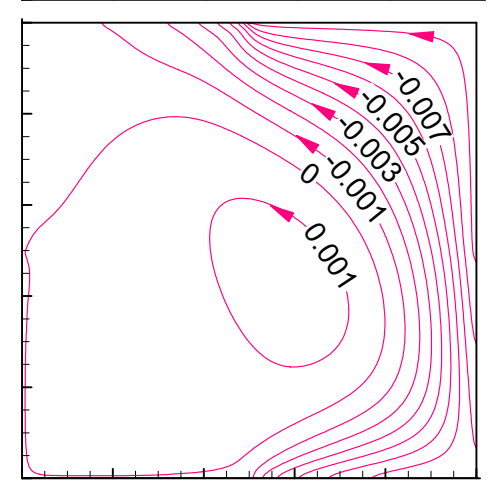

(b) Partially driven cavity

Fig. 2 (Color online) Impact of Grashof number $\left(\mathrm{Gr}=10^{4}-10^{6}\right)$ at $\mathrm{Ha}=30, \mathrm{Da}=10^{-4}$ and $\varepsilon=0.6$ on thermofluid flow structures in stationary cavity (a) and partially driven cavity with $\mathrm{Re}=500$ and $v_{w}=-1$ (b). [Blue lines-streamlines, dashed red lines-isotherms and red lines_-heatlines] 
increases with increasing $\mathrm{Gr}$. Of course, the increase in $\mathrm{Nu}$ with $\mathrm{Gr}$ indicates that the buoyancy is a dominant factor even with the presence of magnetic field.

The aforementioned observations (of Fig. 2a, stationary cavity) are severely modified due to the partial sliding motion (PDC) about the left-top corner at $\mathrm{Re}=500$ and $v_{w}=-1$ as shown in Fig. 2b. The overall flow as well as the heat transport process is governed by the combined effect of shear flow and buoyant flow in the presence of magnetic field. The partial sliding motion induces a shear flow in counter-clockwise (CCW) direction. It favors and strengthens the buoyancy-induced circulation (which is also in the same CCW direction). Interestingly, the core of fluid circulation takes 'triangular' shape for PDC at $\mathrm{Gr} \leq 10^{5}$ and is grossly shifted from the center of stationary cavity to the top-left corner of PDC. It is also expected from the fundamental viewpoint that the buoyancy-induced MHD circulation is aided by the shear flow induced by partial wall motion. The streamlines in all the snapshots indicate a single prominent circulation in counter-clockwise (CCW) direction. The value of the maximum streamfunction $|\psi|_{\text {max }}$ (as indicated by the contours of streamlines) increases as Gr increases, resulting in stronger flow velocity (indicated by more number of streamlines). The isotherms are more deflected following the direction of flow. At $\mathrm{Gr}=10^{6}$, the individual patterns of isotherms and heatlines of stationary cavity and PDC are found more similar compared to other results at $\mathrm{Gr} \leq 10^{5}$. As $\mathrm{Gr}$ value increases to $10^{6}$, the temperature gradient at the corner heater increases-this results in clustering of isotherms near the heated wall. Thus, the isotherms of higher contour value are distorted toward the heated corner at the right side, whereas the isotherms of lower contour value distorted toward the cold corner at the left side. With the increasing $\mathrm{Gr}$ value, as velocity increases, the more or less straight, diagonal isotherms at $\mathrm{Gr} \leq 10^{5}$ become almost vertical or ' $\mathrm{S}$ '-like shape at $\mathrm{Gr}=10^{6}$. The heatlines, which address both conductive and convective fluxes collectively, are oriented between the corner heater and the corner cooler. A large energy circulation is developed at $\mathrm{Gr}=10^{6}$, and the transport corridors of thermal energy are distorted. Through heatlines, it is clear that the major heat energy from the bottom corner source is transported to the top partially moving wall. From the Nu values of both the cases of stationary cavity and partially driven cavity, it is clear that the heat transfer substantially increases in the case of PDC for all the values of Gr. For the present condition of $\operatorname{Re}=500$ and $v_{w}=-1$, with respect to the stationary cavity, the order of enhanced heat transfer for PDC is $30-40 \%$. All these results discussed here clearly indicate the efficacy of partially driven cavity (PDC) to enhance the heat transfer and to modify the flow structures. With the establishment of advantage of thermal performance of PDC, the effects of influencing coupled physics-related parameters ( $\mathrm{Ri}, \mathrm{Re}$, $\mathrm{Ha}, \mathrm{Da}, \varepsilon)$ on the local distributions as well as on the global performance are discussed systematically in the following subsections.

\subsection{Impact of operating flow regimes (Ri)}

Conventionally a system can operate in the regimes of forced, mixed or free convection. To address these flow regimes, the values of Richardson number are chosen as $\mathrm{Ri}=0.1,1$ and 100 for forced, mixed and free convection, respectively. The corresponding results are shown in Fig. 3, keeping other involved parameters, $\operatorname{Re}=200$, $\mathrm{Ha}=30, \mathrm{Da}=10^{-4}$ and $\varepsilon=0.6$, fixed. Furthermore, the directional impact of partial sliding (of the adjacent walls) is also captured by setting velocity of the walls at $v_{w}= \pm 1$ [for clockwise (CW) and counter-clockwise (CCW) partial motion, respectively] as indicated in Fig. 3a, b. The flow regimes and the wall-moving directions markedly affect the thermohydrodynamics in the cavity. Under forced convection (at $R i=0.1$ ) and mixed convection $(R i=1)$, the heat transfer of PDC is more or less the same irrespective of CCW or CW motion. At smaller values of Ri (with fixed $\operatorname{Re}=200$ ), the weakly developed buoyant flow is principally dominated by forced convection (due to moving lids). This can be realized from the streamline contours at $\mathrm{Ri} \leq 1$ for both $v_{w}= \pm 1$. However, at $\mathrm{Ri}=1 \mathrm{in} \mathrm{Fig.} 3 \mathrm{a}$, the existence of tiny vortex near the right-bottom corner is observed due to counter-rotating buoyant flow (against shear-induced forced circulation). For the low Ri values, the isotherms are distributed diagonally (like slash '/') in the cavity from the heating corner to the cooling corner. Obviously, the orientation of contours is modified by the direction of fluid circulations (CCW and CW) in the cavity. At weak buoyancy effect $(\mathrm{Ri} \leq 1)$, the heat energy can flow in a direct manner from the source to the sink apparently diagonally (backslash ' $\prime^{\prime}$ ). The direction of partial sliding has marked impact on the distribution of heatlines.

With the increase in Richardson number (as Re is fixed) the buoyant flow (induced by corner heater) becomes stronger, resulting in a significant change at $\mathrm{Ri}=100$ (Fig. 3a, b). In Fig. 3 a with positive wall velocity, $v_{w}=+1$, the streamlines indicate a significant reduction of shear flow vortex at the top-left corner. The strong circulation exists in CCW direction with an elliptical circulation core. The same is applicable for negative wall velocity $\left(v_{w}=+1\right)$ in Fig. 3b. However, here due to aiding effect (of natural and forced convection), a single circulation with comparatively higher $\psi_{\max }$ is observed. It results into relatively higher $\mathrm{Nu}$ ( 80.18\%) compared to the lower Ri value of 0.1 .

For both the cases of $v_{w}= \pm 1$, the isotherms are distorted about the active corners resulting in a ' $S$ '-like shape of contours. Like fluid flow, the heat flow also takes place 

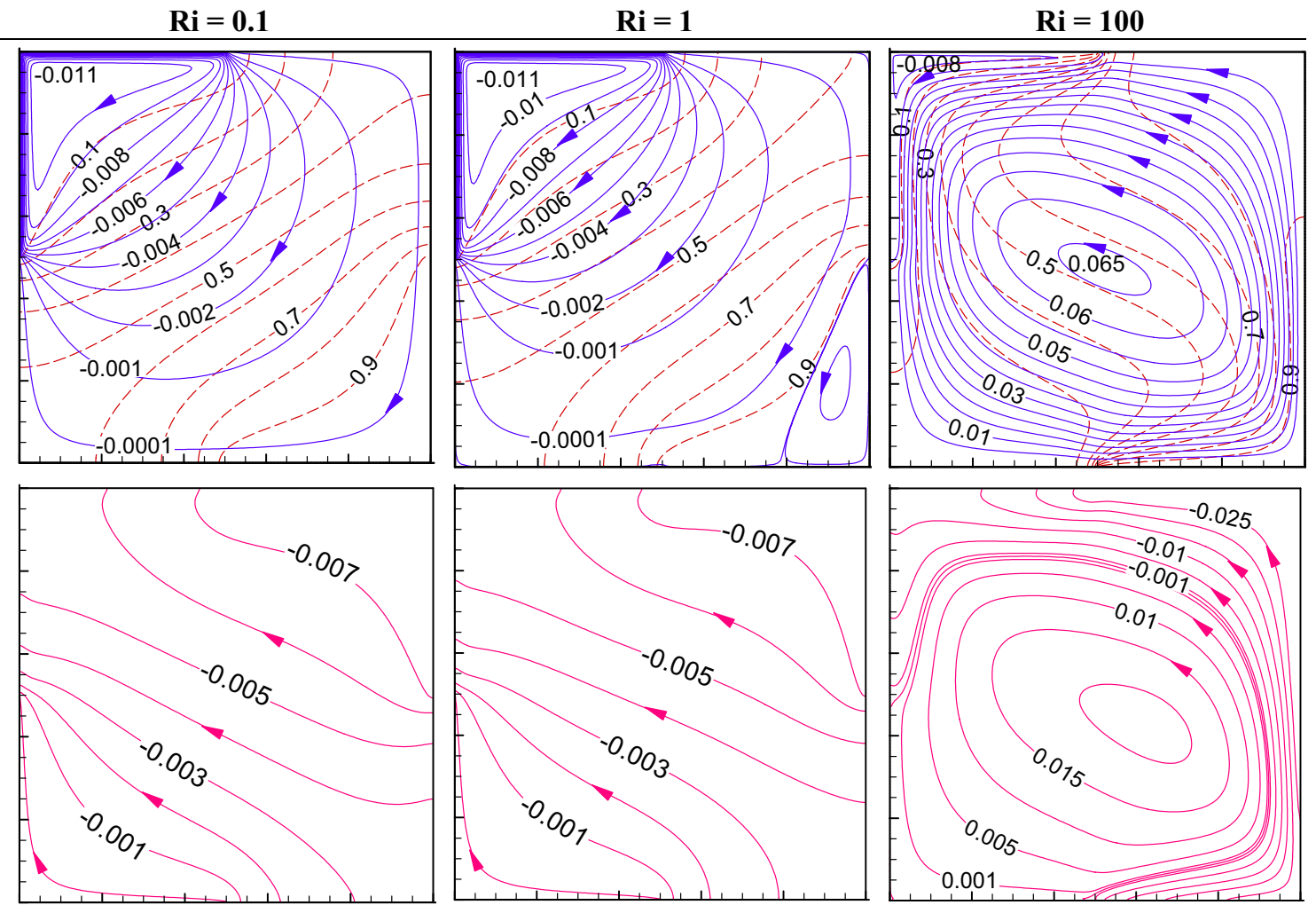

(a)

$\mathrm{Nu}=\mathbf{1 . 0 6 9}$

$\mathrm{Nu}=\mathbf{1 . 0 5 4}$

$\mathrm{Nu}=4.386$
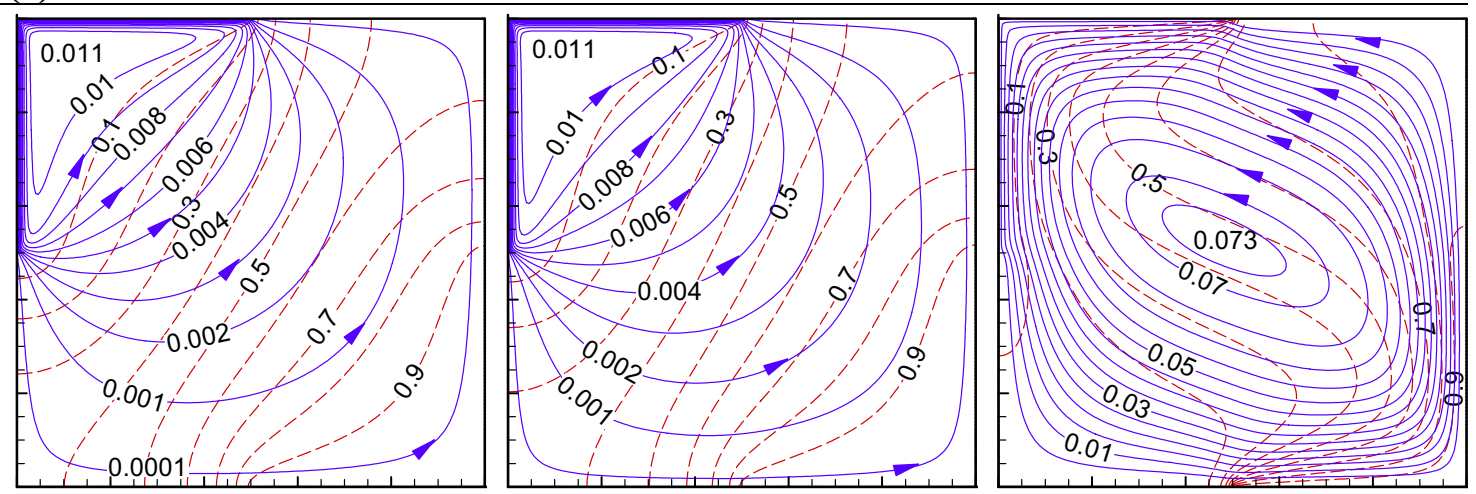

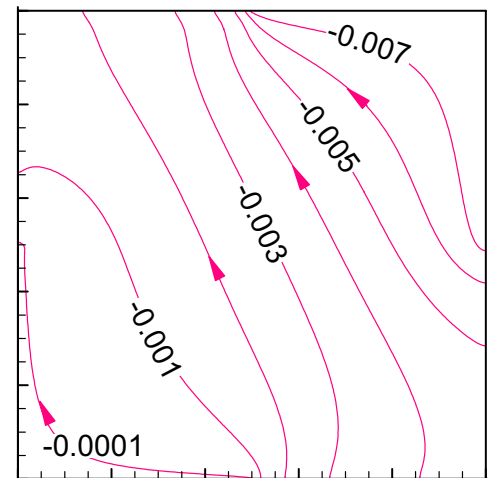

(b)

$\mathrm{Nu}=\mathbf{1 . 0 7 2}$

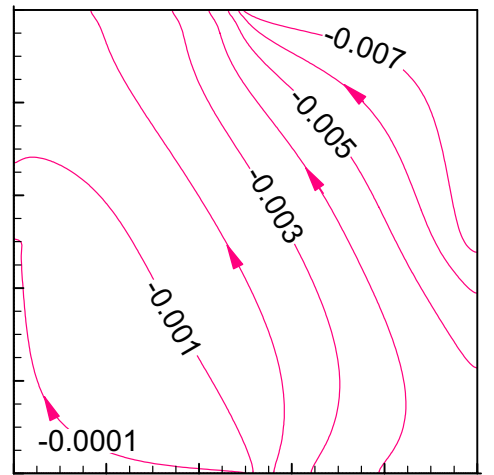

$\mathrm{Nu}=1.099$

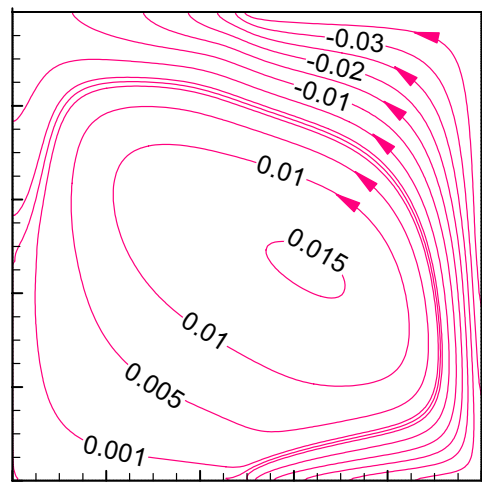

$\mathrm{Nu}=\mathbf{5 . 4 0 8}$
Fig. 3 (Color online) Impact of Richardson number (Ri) on fluid flow and thermal behavior at $\mathrm{Re}=200, \mathrm{Ha}=30, \mathrm{Da}=10^{-4}$ and $\varepsilon=0.6$ for $v_{w}=+1$ (a) and $v_{w}=-1$ (b). Unless specified, the contour intervals for isotherms are chosen as 0.1 ; for streamlines and heatlines inter- vals are chosen as $0.001,0.001$ (first two columns) and 0.005, 0.005 (third column), respectively. [Blue lines-streamlines, dashed red lines-isotherms and red lines-heatlines] 
in CCW, resulting in a large energy circulation. The plot of heatlines clearly shows the energy transport corridors from the heating corner to the cooling corner. The heat energy carrying contour lines (created from heated corner) rises toward the upper side of the cavity; after that, it reaches the cold corner walls and finally heat energy is rejected out. In regard to heat transfer, it is seen that $\mathrm{Nu}$ is almost the same for both the cases of lid motions (clockwise and anti-clockwise) when $\mathrm{Ri}=0.1$ and 1 . However, at higher $\mathrm{Ri}=100$, a significant difference in the values of $\mathrm{Nu}$ (23.3\% enhanced under CCW motion) is observed. In general, the counter-clockwise lid motion is found more effective over the clockwise lid motion. CCW motion favors natural convection and acts as assisting flow.

\subsection{Impact of partial sliding speed (Re)}

The influence of partial sliding motion on the thermohydrodynamics is investigated utilizing different moving speeds of the active cold walls in Fig. 4. For the same fluid and geometry, the different speeds are imposed through the Reynolds number, $R e=10,200$ and 500. Both positive velocity and negative velocity of the walls $v_{w}= \pm 1$ are considered and shown in Fig. 4a, b, respectively. Other conditions are taken at $\mathrm{Ri}=10, \mathrm{Ha}=30, \mathrm{Da}=10^{-4}$ and $\varepsilon=0.6$, fixed. At the lower value of $\operatorname{Re}=10$, the flow structure is dominated by the forced convection induced from the partial translation. Corresponding heat transfer is dominated by thermal conduction (as corresponding $\mathrm{Gr}=10^{3}$ ). This is evident from the distortion-free isotherm contours which are distributed in a straightway following the shape of the active parts of the walls. The energy transport through the heatlines happens straightforward from the heated corner to the cold corner.

However, at an increased Reynolds number, $R e=200$ and 500, the buoyancy-induced flow becomes stronger (as $\mathrm{Ri}$ is fixed at 10). The existence of traditional opposing (Fig. 4a) and aiding (Fig. 4b) flow scenarios appears in the corner-heated PDC. Two vortices, shear driven and buoyancy induced, are clearly located in the cavity in Fig. 4a. As Re increases, Gr increases by the square of Re. It results in relatively stronger natural convection circulation; thus, shear-driven vortex at the top-left corner shrinks at $\operatorname{Re}=500$. The aiding flow is clearly reflected in Fig. $4 \mathrm{~b}$ with the same sense of rotations and, thus, from the combined effect forms a single large circulation covering entire cavity. With fixed $\mathrm{Ri}$, as Re affects both wall-moving speed and $\mathrm{Gr}$, the higher Re value severely affects all the flow structures-streamlines, isotherms and heatlines. The energy circulations in heatlines plots are present for both $v_{w}= \pm 1$, which covers the major portion of the cavity. Thus, heat flows (from the heater to the cooler) through the long paths. The values of $\mathrm{Nu}$ indicate heat transfer is significantly amplified at higher $\operatorname{Re}$ and $v_{\mathrm{w}}=-1$.

\subsection{Influence of Darcy number (Da)}

The Darcy number ( $\mathrm{Da}$ ) of the porous medium has strong impact on the thermofluid flow structures. It is explored in Fig. 5 for the proposed PDC at $\mathrm{Re}=200, \mathrm{Ri}=10, \mathrm{Ha}=30$, $\varepsilon=0.6$ and $v_{w}= \pm 1$. The outcome of partial motion is clearly observed at lower Da value $\left(10^{-7}\right)$. As the shear-driven circulation takes up around $60 \%$ of the space inside the enclosure in Fig. 5a, the buoyancy-driven vortex is formed about the right-bottom corner of the cavity. The contours of isotherms are allocated symmetrically about the two corners of the enclosure. However, when Da increases to $10^{-5}$, the buoyancy-driven circulation occupies more than $60 \%$ of the cavity space compared to the lower Da value $\left(10^{-7}\right)$. This happens so as the flow passage becomes less flow resistive due to improved permeability of porous medium at an enhanced $\mathrm{Da}$. The convective fluid-flow velocity becomes stronger. It becomes substantially high at $\mathrm{Da}=10^{-3}$, which is evident from the corresponding plots of streamlines. It is also important to note that the allotments of isotherms at $\mathrm{Da}=10^{-7}$ and $10^{-5}$ are almost comparable. However, it significantly differs at $\mathrm{Da}=10^{-3}$. As the patterns of streamlines at $\mathrm{Da} \leq 10^{-5}$ are alike, the similar manifestations in the cases of isotherms and heatlines are observed at $\mathrm{Da} \leq 10^{-5}$. However, at higher $\mathrm{Da}=10^{-3}$, the strong non-uniformity is noted in the distribution of isotherms and heatlines, and energy circulation cells are formed. Furthermore, the direction of partial sliding, $v_{w}= \pm 1$, severely affects the interaction shear flow and buoyant flow as realizable in Fig. $5 \mathrm{a}$ for $v_{\mathrm{w}}=+1$ (opposing or counter-acting flow situation) and Fig. $5 b$ for $v_{w}=-1$ (assisting or aiding flow situation). Two vortices developed in the cavity under $v_{w}=+1$ in Fig. 5a show different strengths and sizes depending upon the Da value. However, when $v_{w}=-1$ in Fig. $5 \mathrm{~b}$, the cavity is fully occupied by a single CCW circulation. Secondly, the marked increase in $\mathrm{Nu}$ is observed at $v_{w}=-1$ due to a favorable adding effect of both shear flow and buoyant flow, and the significant change in distribution pattern of heatlines is observed at $\mathrm{Da}=10^{-3}$.

\subsection{Influence of porosity $(\varepsilon)$}

The effect of porosity $(\varepsilon)$ on thermal performance of PDC is illustrated in Fig. 6 keeping other parameters $(R e=200$, $\mathrm{Ri}=10, \mathrm{Ha}=30, \mathrm{Da}=10^{-4}$ and $v_{w}= \pm 1$ ) constant. Both CW and CCW motion of partial sliding are considered in Fig. 6a, b. The results show an augmented thermofluid feature in the cavity with the increase in porosity value. Under the opposing flow (Fig. 6a), as the value of $\varepsilon$ increases, the 

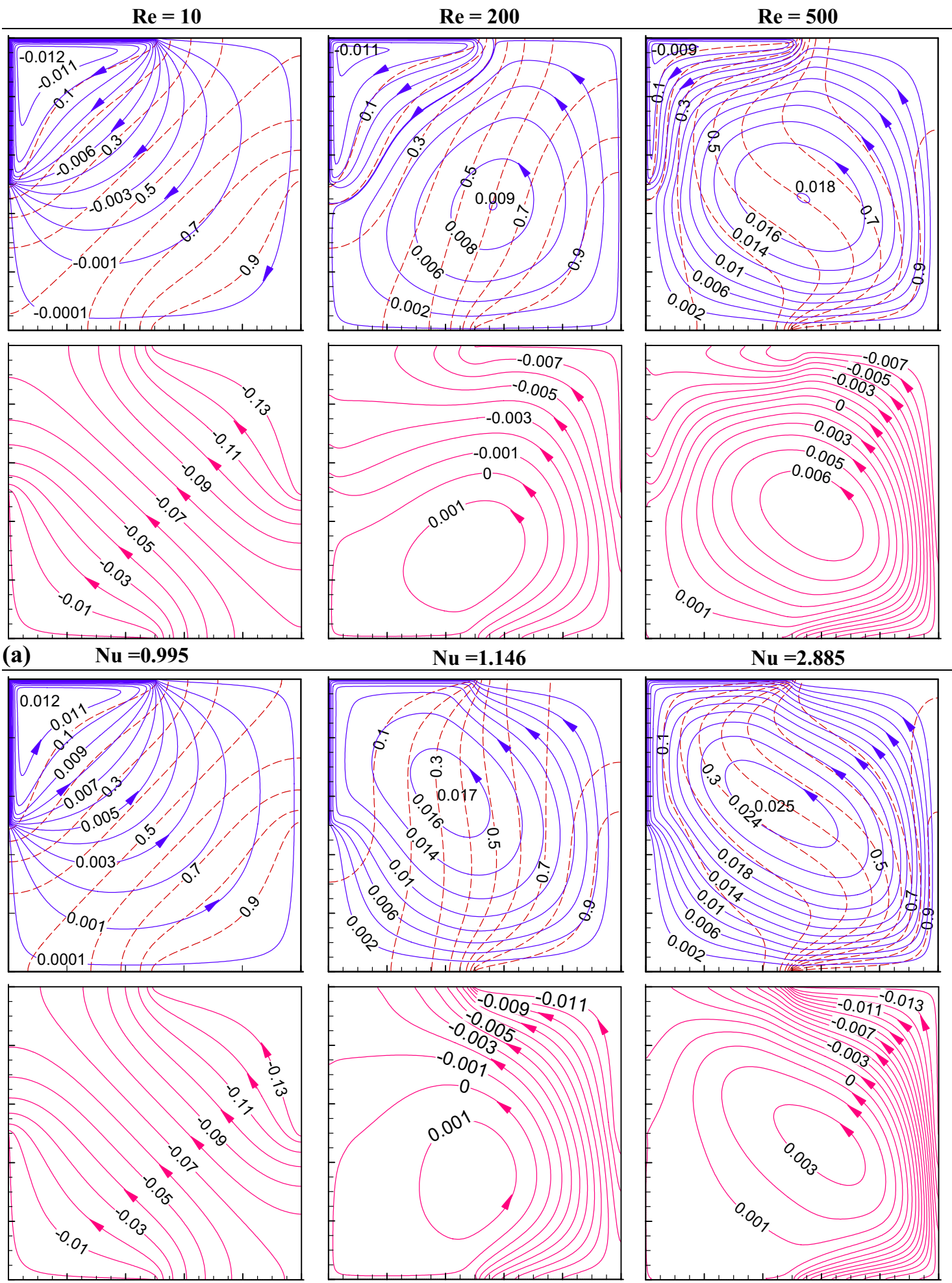

(b)

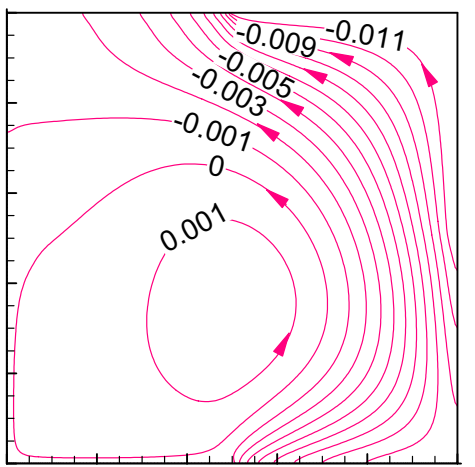

$\mathrm{Nu}=\mathbf{1 . 7 0 2}$

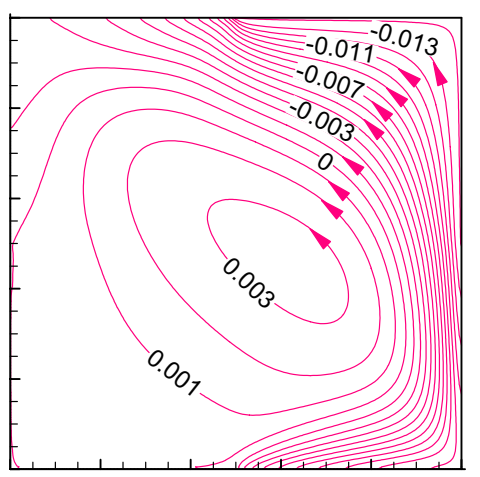

$\mathrm{Nu}=\mathbf{5 . 0 0 0}$

Fig. 4 (Color online) Impact of Reynolds number (Re) on fluid flow and thermal behavior at $\mathrm{Ri}=10, \mathrm{Ha}=30, \mathrm{Da}=10^{-4}$ and $\varepsilon=0.6$ for $v_{w}=+1$ (a) and $v_{w}=-1$ (b). Unless specified, the contour intervals for isotherms are chosen as 0.1 ; for streamlines and heatlines inter- vals are chosen as $0.001,0.01$ (first column) and 0.002, 0.001 (last two columns), respectively. [Blue lines-streamlines, dashed red lines-isotherms and red lines_-heatlines] 

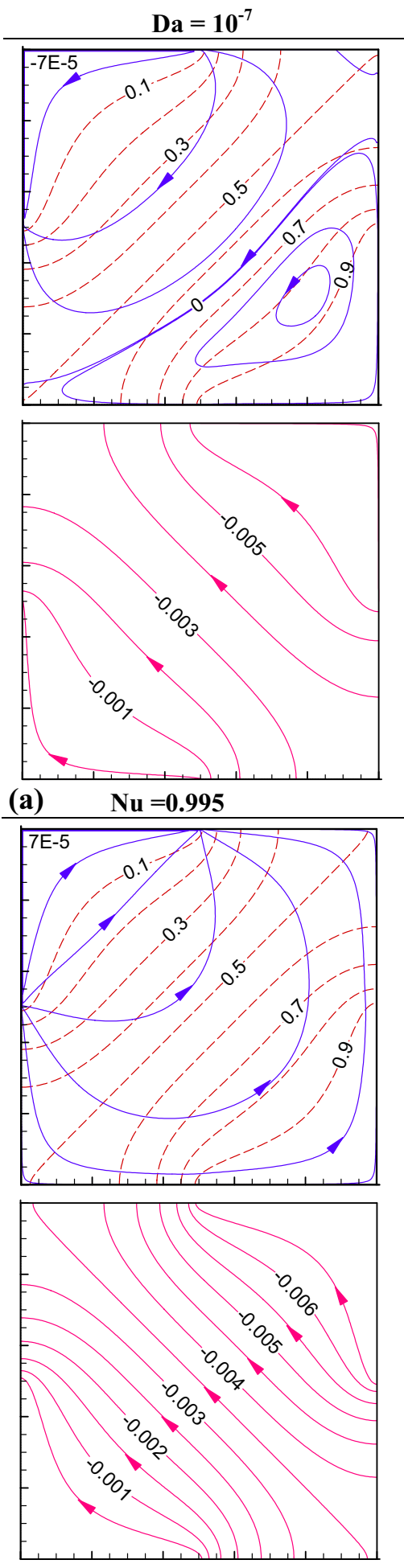

(b)
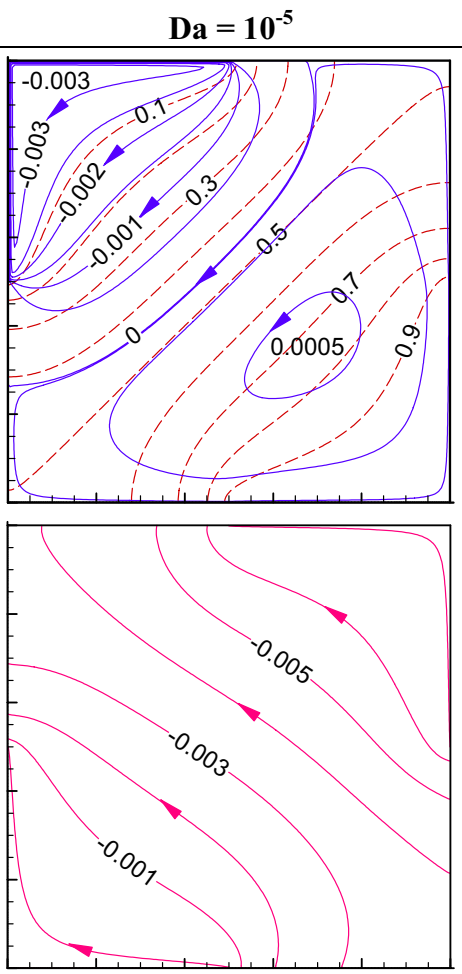

$\mathrm{Nu}=\mathbf{0 . 9 9 9}$
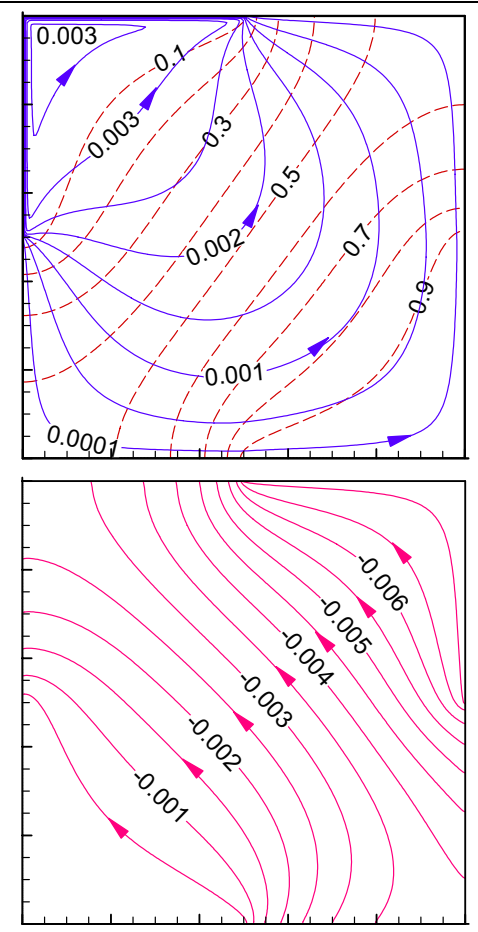

$\mathrm{Nu}=\mathbf{1 . 0 1 6}$
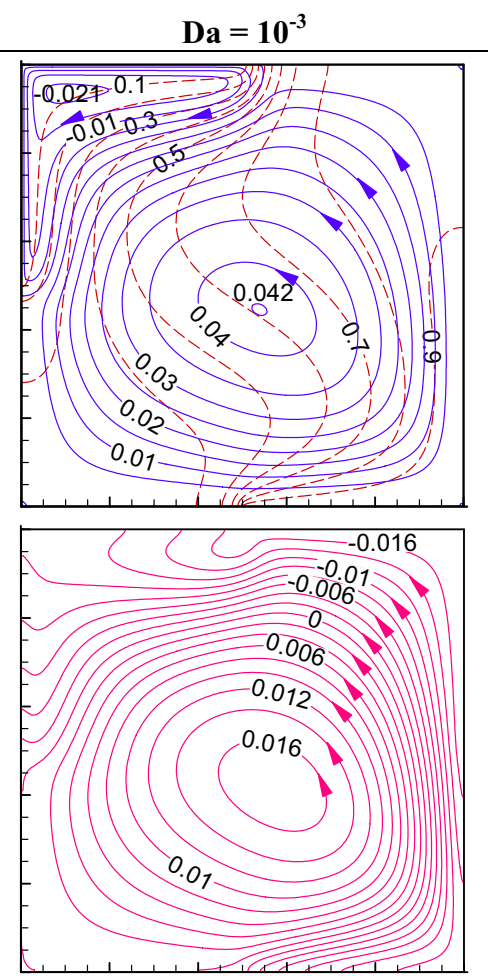

(a) $\mathrm{Nu}=\mathbf{2 . 4 5 0}$
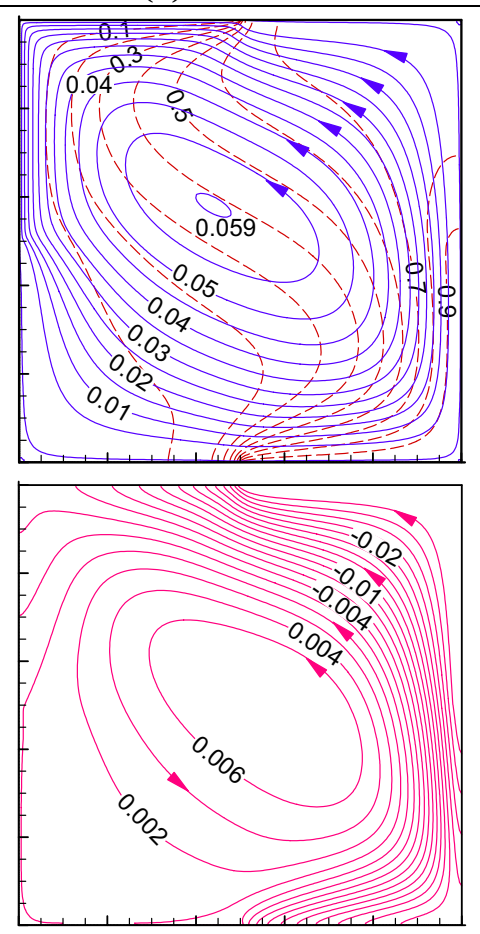

(b) $\mathrm{Nu}=4.337$
Fig. 5 (Color online) Impact of Darcy number (Da) on fluid flow and thermal behavior at $\mathrm{Ri}=10, \mathrm{Re}=200, \mathrm{Ha}=30$ and $\varepsilon=0.6$ for $v_{w}=+1$ (a) and $v_{w}=-1$ (b). Unless specified, the contour intervals for isotherms are chosen as 0.1 ; for streamlines and heatlines intervals are chosen as randomly, 0.001 (first column), 0.0005, 0.001 (second column) and $0.005,0.002$ (third column), respectively. [Blue linesstreamlines, dashed red lines-isotherms and red lines-heatlines] 

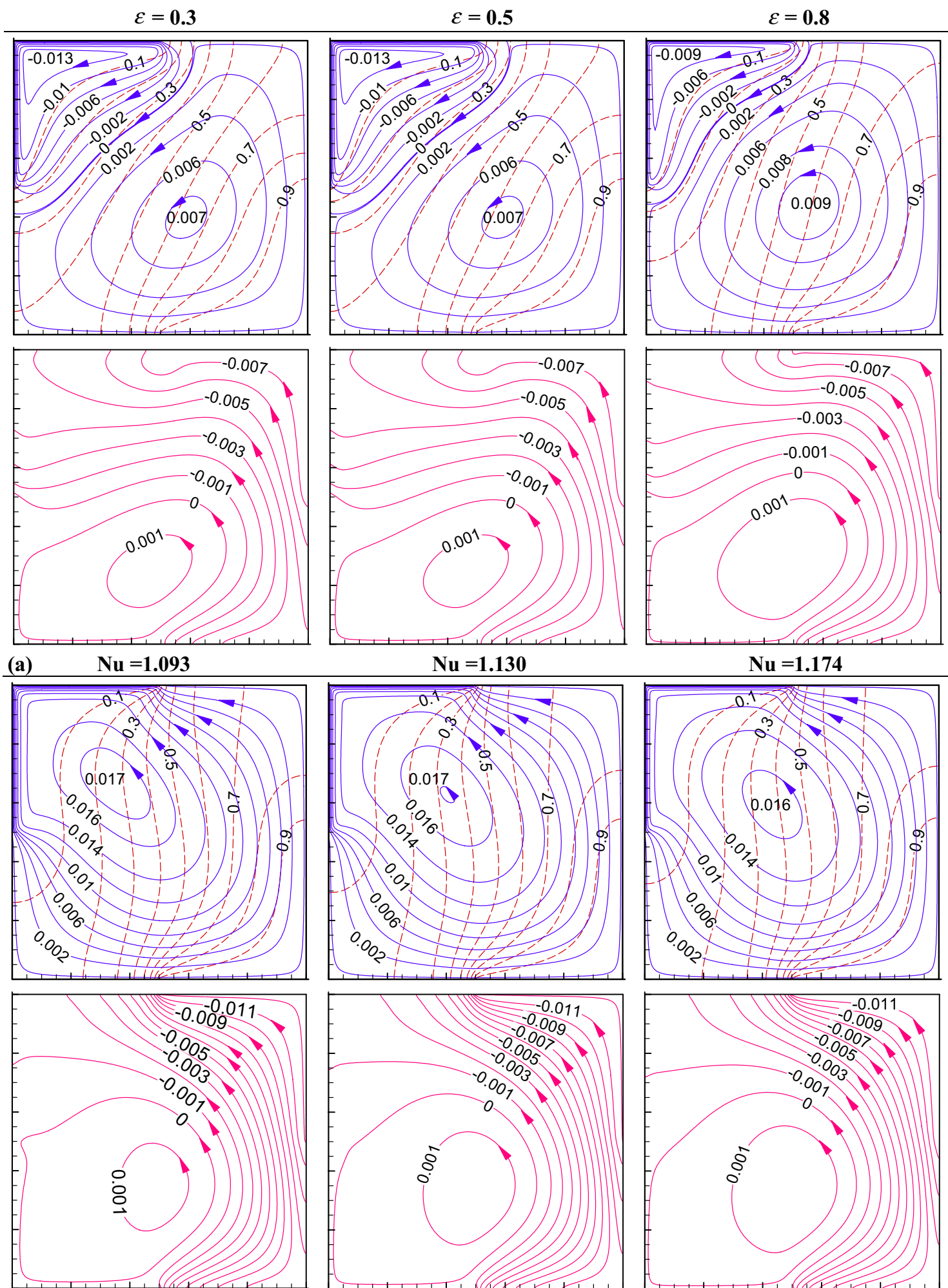

(b)

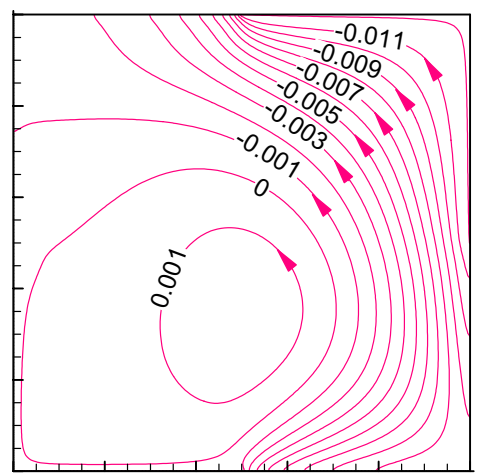

$\mathrm{Nu}=\mathbf{1 . 6 9 5}$

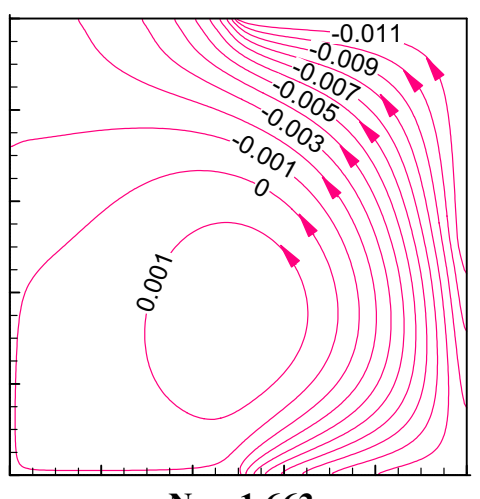

Fig. 6 (Color online) Porosity $(\varepsilon)$ effect at $\mathrm{Ri}=10, \mathrm{Re}=200, \mathrm{Ha}=30$ and $\mathrm{Da}=10^{-4}$ on thermofluid flow structures in PDC with $v_{w}=+1$ (a) and $v_{w}=-1$ (b). Unless specified, the contour intervals of isotherms, streamlines and heatlines plots are chosen as 0.1, 0.002 and 0.001 , respectively. [Blue lines-streamlines, dashed red lines-isotherms and red lines-heatlines] 
buoyancy-dominated circulation marginally gains its strength by reducing the strength of shear-induced circulation. Overall, a minor change is noted in the patterns of streamlines, isotherms and heatlines for both the boundary velocities $\left(v_{w}= \pm 1\right)$. This leads to marginal increase in $\mathrm{Nu}$ value (as indicated below the plots of heatlines) with increasing porosity. This happens so, as the porosity of the porous domain increases, the fluid flow encounters lesser resistance.

\subsection{Impact of Hartmann number (Ha)}

For the present problem, the external uniform magnetic field (as shown in Fig. 1) is applied horizontally, which effect is simulated through Hartmann number $(\mathrm{Ha})$. From governing Eq. (3), it is clear that the magnetic force dampens the vertical component $(V$ ) of velocity. Due to the couple effects among the different forces (magnetic, buoyancy and shear forces), the Hartmann number $(\mathrm{Ha})$ severely influences thermofluid flow dynamics in the cavity. It can be realized in Fig. 7 with the three values of $\mathrm{Ha}=0,50,100$ keeping other parameters fixed $(\mathrm{Ri}=10$, $\operatorname{Re}=200, \mathrm{Da}=10^{-4}, \varepsilon=0.6$ and $v_{w}= \pm 1$ ). To understand the impact of magnetic field on the streamlines, isotherms, heatlines and $\mathrm{Nu}$, a case of no-magnetic field $(\mathrm{Ha}=0)$ is presented in the first column. For $\mathrm{Ha}=0$ with $v_{w}=+1$ as in Fig. 7a, the buoyant flow (CCW) dominates the shear flow (CW) by a bigger circulation occupying most of the space of PDC. The strength of the natural convection is substantially high at $\mathrm{Re}=200$ and $\mathrm{Ri}=10$ (resulting in $\mathrm{Gr}=4 \times 10^{5}$ ). These two unequal flow vortices originate as a result of mixed convection evolved due to partial sliding of the corner walls (forced convection) and differential heating and cooling at the corners of the cavity (natural convection from buoyancy-induced flow). The contours of corresponding isotherms are distributed throughout the cavity depending upon the thermal gradient in between two active corners. The contours of heatlines clearly indicate about the presence of energy recirculating vortex in the lower half of the cavity. The thermal energy is transported from the heat source toward the heat sink following the rotation of the energy circulation vortex.

As the Hartmann number increases to $\mathrm{Ha}=50$ or 100 , though the overall patterns of streamlines, isotherms and heatlines are similar to the earlier patterns at $\mathrm{Ha}=0$, the buoyancy-induced circulation weakens (as established from the values of the maximum streamfunction). As a consequence of weakening buoyant circulation, the shear flow vortex marginally gains its strength at higher $\mathrm{Ha}$. The heat transfer in terms of average Nusselt number is found maximum at $\mathrm{Ha}=0$ (in the absence of magnetic field). The direction of partial sliding motion has substantial impact on the magnetothermofluid flow. Though the trend of $\mathrm{Nu}$ with Ha remains unaltered, the magnitude of $\mathrm{Nu}$ is significantly high with the negative moving velocity $\left(v_{w}=-1\right.$, in Fig. 7b). In general, irrespective of the moving direction of the walls, with the increase in Ha values from 0 to 100, the significant reduction in the energy re-circulation cells is observed from the heatlines plots. The temperature distribution in the cavity is distorted as could be seen from the isotherm plots.

\subsection{Heat transfer characteristics of PDC}

The heat transfer characteristics of the partially driven cavity are summarized under different parametric variations of $\mathrm{Re}, \varepsilon, \mathrm{Da}$ and $\mathrm{Ha}$ for $\mathrm{Ri}=0.1-100$ and $v_{w}= \pm 1$ in Fig. 8. The fixed parameters are taken as $\mathrm{Re}=200, \mathrm{Ha}=30, \mathrm{Da}=10^{-4}$ and $\varepsilon=0.6$. Heat transfer characteristics are assessed in terms of the average Nusselt number Nu as given in Eq. (6) for the cold active walls.

In Fig. 8a, Reynolds number variation is illustrated for a range of $R e=10$ to 500 . At $R i \leq 1$, there is almost no effect of Re on Nu. However, at $\mathrm{Ri}=10$ or above, Nu substantially increases with Re. Higher Ri has greater impact on the heat transfer. The figure clearly indicates that Nu value increases monotonically with the increase in Reynolds number for both the directions of partial sliding $\left(v_{w}= \pm 1\right)$. At higher Reynolds number $\operatorname{Re}>200$, the average heat removal rate is higher. The increment of average Nu is much higher for $\mathrm{Ri}>10$. Furthermore, the direction of partial sliding plays an important role on the thermal performance. The figure suggests the velocity $v_{w}=-1$ is always a better choice for higher heat transfer rate. The negative velocity $\left(v_{w}=-1\right)$ compared to the positive velocity $\left(v_{w}=+1\right)$ results in an enhanced heat transfer for all Re values. The Nu difference of two velocities $v_{w}= \pm 1$ of the active cold walls grows continually as $R e$ increases. The reason of such increment is due to the fact that as Re increases (keeping all other parameters at fixed value), corresponding Grashof number increases from $10^{2}$ to $2.5 \times 10^{6}$, leading to a significant increase in buoyancy effect (which is also evident from Fig. 4).

As observed above (Fig. 8a), the porosity impact is clearly revealed in Fig. $8 \mathrm{~b}$ with $\mathrm{Ri}=100$ and $\mathrm{Ri}=10$ (only for negative velocity $v_{w}=-1$ ). The reason for such increment is due to the fact that as porosity increases (keeping all other parameters at fixed value), the resistance to fluid motion also decreases. At higher value of $\mathrm{Ri}=100$, the buoyancy effect increases significantly, leading to an increase in fluid motion. This effect is also further influenced by the direction of partial sliding. It is also observed that Nu value is almost independent of increase in porosity when $\mathrm{Ri} \leq 10$, for any direction of wall moving.

The effect of increasing Darcy number on the average Nusselt number is presented in Fig. $8 \mathrm{c}$. It is observed 

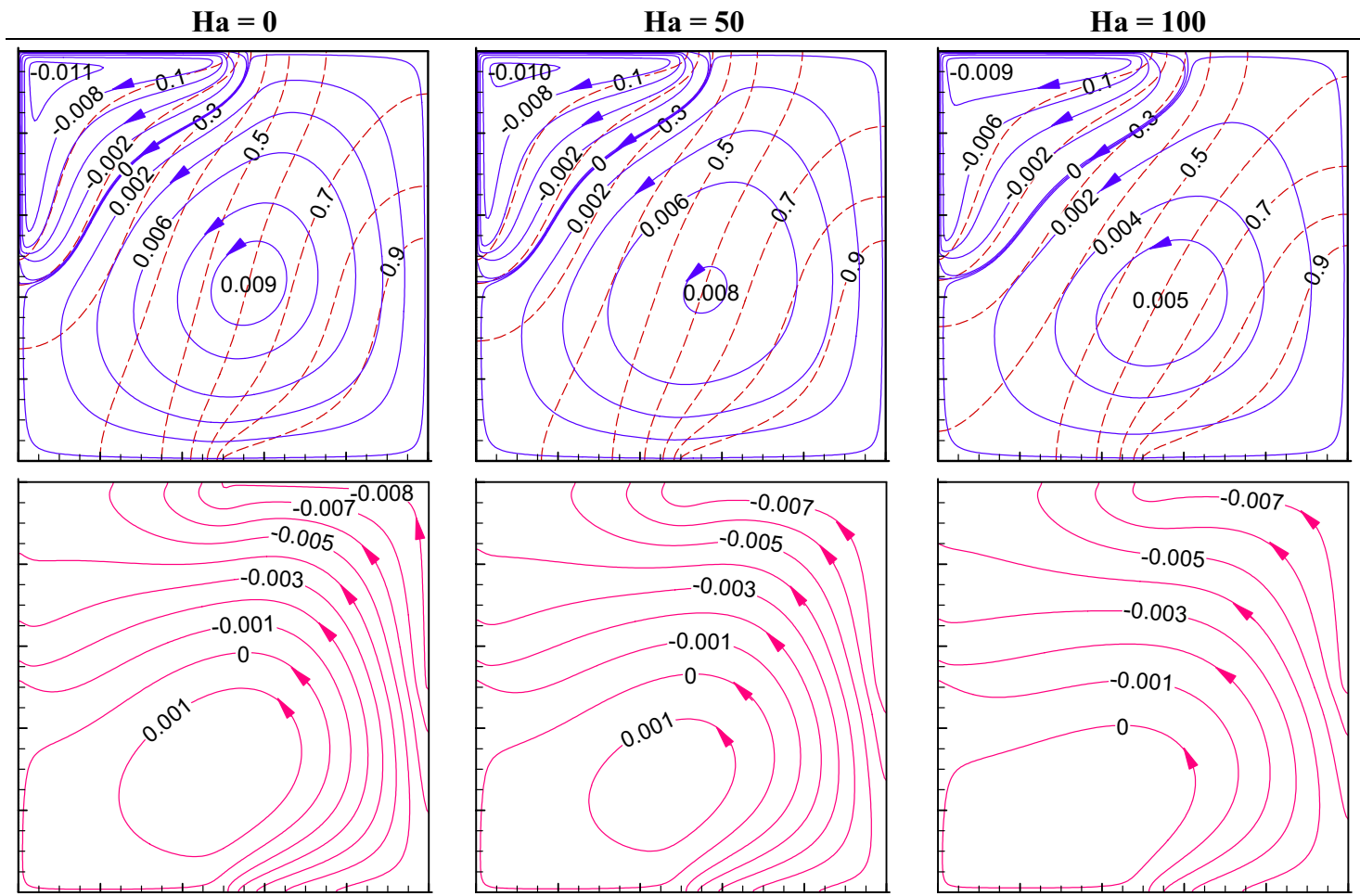

(a)
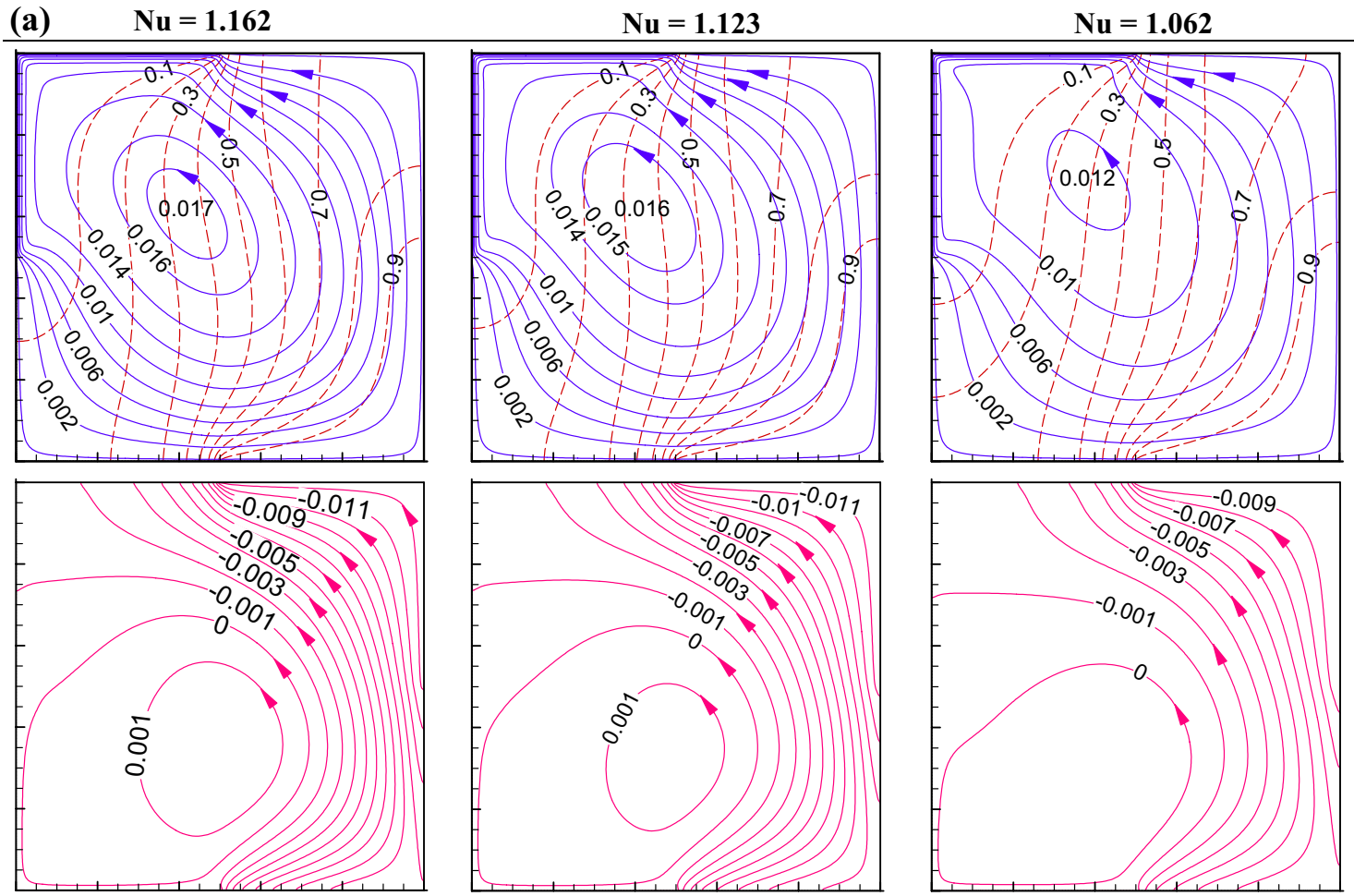

(b)

$\mathrm{Nu}=\mathbf{1 . 7 4 3}$

$\mathrm{Nu}=\mathbf{1 . 6 3 6}$

$\mathbf{N u}=\mathbf{1 . 4 1 7}$

Fig. 7 (Color online) Impact of Hartmann number $(\mathrm{Ha})$ on fluid flow and thermal behavior at $\mathrm{Ri}=10, \mathrm{Re}=200, \mathrm{Da}=10^{-4}, \varepsilon=0.6$ for $v_{w}=+1$ (a) and $v_{w}=-1$ (b). Unless specified, the contour intervals of isotherms, streamlines and heatlines plots are chosen as 0.1, 0.002 and 0.001 , respectively. [Blue lines-streamlines, dashed red lines-isotherms and red lines_-heatlines] 

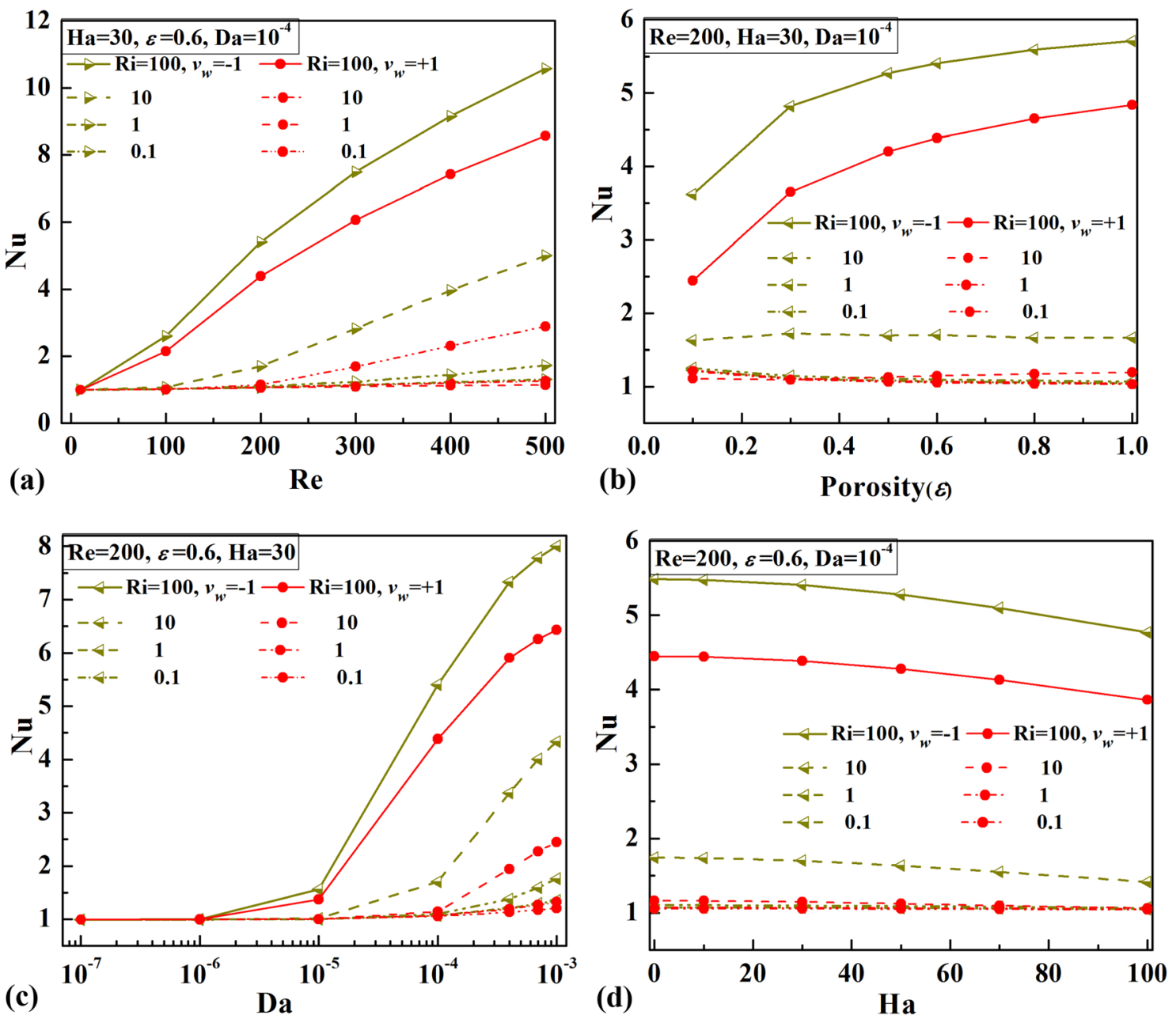

Fig. 8 (Color online) Heat transfer characteristics under parametric conditions of Re (a), $\varepsilon$ (b), Da (c) and Ha (d) keeping other parameters fixed at $\mathrm{Re}=200, \mathrm{Ha}=30, \mathrm{Da}=10^{-4}$ and $\varepsilon=0.6$

that there is no influence of $\mathrm{Da} \leq 10^{-6}$ on the overall heat transfer process. However, the heat transfer rate increases significantly at $\mathrm{Da}>10^{-5}$ for any direction of partial sliding. As the Da increases (keeping all other parameters at fixed value), the resistance to fluid flow of the porous medium reduces, leading to increased heat transfer. This effect is also further influenced by the direction of partial translation.

Magnetic effect shown in Fig. $8 \mathrm{~d}$ shows a monotonically decreasing trend as the Hartmann number Ha increases. It is observed that, at lower $\mathrm{Ri}<10$, the heat removal process is dictated by conduction mode and the effect of magnetic field is less pronounced. However, at higher value of $\mathrm{Ri}=100$, the average $\mathrm{Nu}$ decreases significantly when Ha increases. Here, the heat transport process is governed by the convection mode, which is further suppressed by the imposed magnetic field. This is an interesting consequence and provides guidance for the selection of the influencing parametric range for achieving a control rate of heat transfer.

\subsection{Assessment of velocity and temperature at mid-plane}

In order to understand the underneath flow physics of MHD convection in PDC, the profiles of dimensionless flow variables $(U, V, \theta)$ are prepared by extracting the relevant data along the mid-planes as indicated in Fig. 9. The parametric effects on the flow variables are illustrated in individual subfigures (Fig. 9a-e) considering the variations in $\mathrm{Ri}, \mathrm{Re}, \mathrm{Da}, \mathrm{Ha}$ and $\varepsilon$, taking other parameters fixed at $\mathrm{Re}=200, \mathrm{Ri}=10, \mathrm{Ha}=30, \mathrm{Da}=10^{-4}, \varepsilon=0.6$ and $v_{w}= \pm 1$. The $U$-velocity is generated along the vertical mid-plane, and the horizontal mid-plane is considered for the $V$-velocity and the temperature $\theta$. The directional effect of wall translation is also included in the figures. 


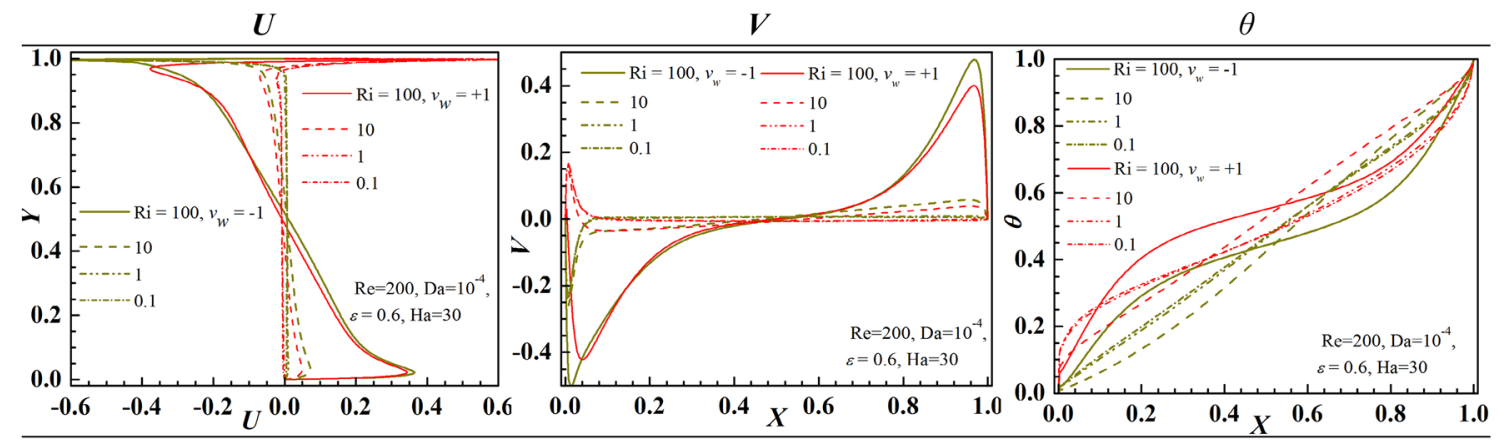

(a)
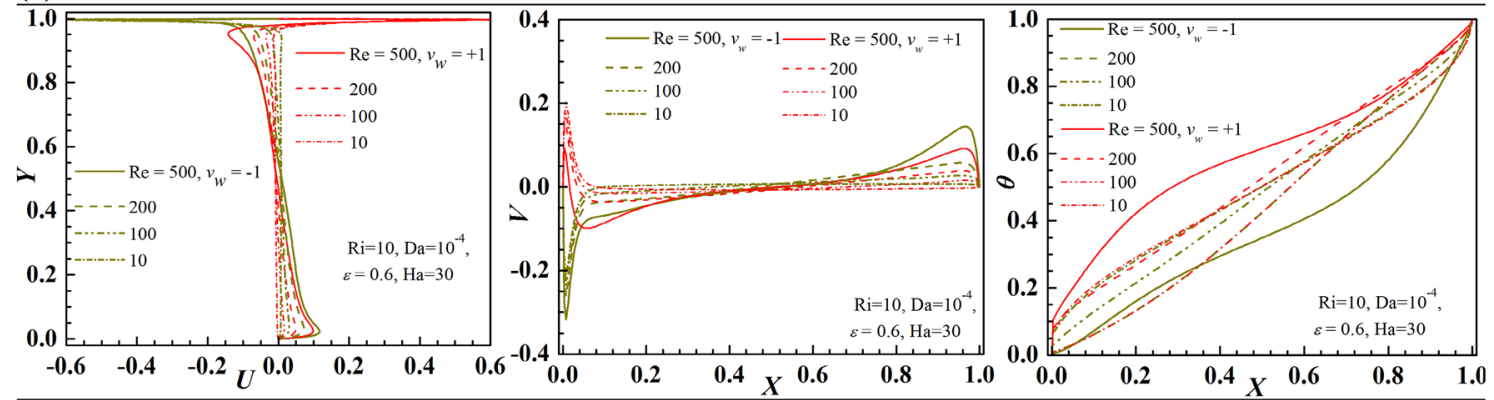

(b)
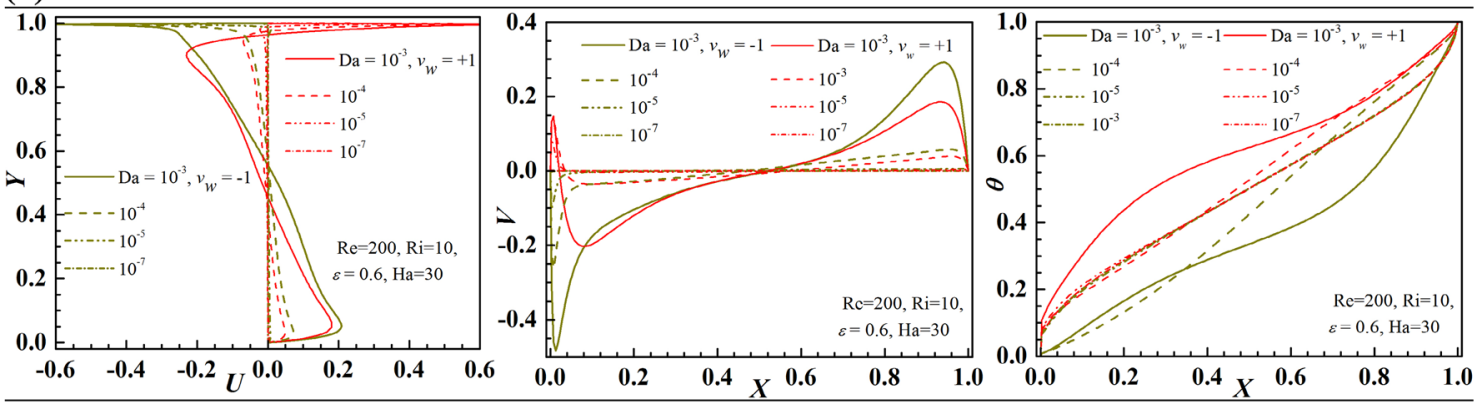

(c)
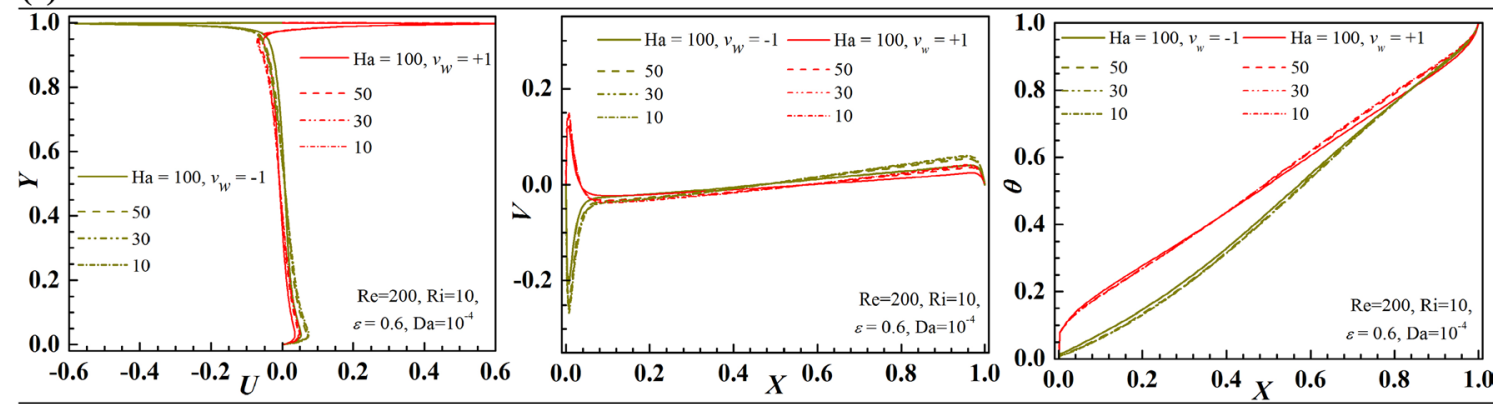

(d)
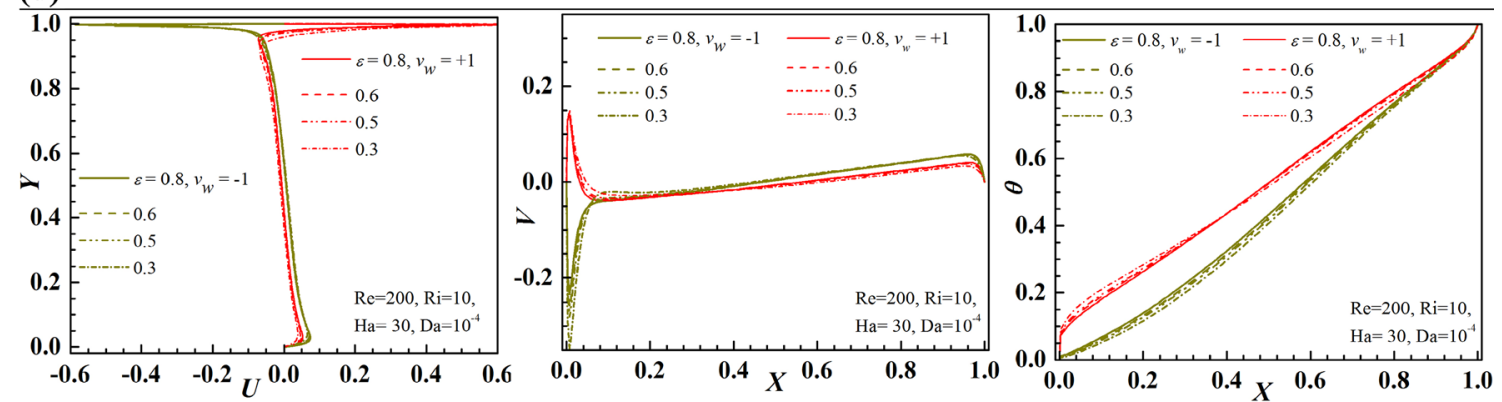

(e)

Fig. 9 (Color online) Mid-plane profiles of velocities $(U, V$ and temperature $(\theta)$ at different $\mathrm{Ri}(\mathbf{a}), \mathrm{Re}(\mathbf{b}), \mathrm{Da}(\mathbf{c}), \mathrm{Ha}(\mathbf{d})$ and $\varepsilon$ (e) keeping other parameters fixed at $\operatorname{Re}=200, \mathrm{Ri}=10, \mathrm{Ha}=30, \mathrm{Da}=10^{-4}, \varepsilon=0.6$ and $v_{w}= \pm 1$ 
The effects of Richardson and Reynolds numbers on $U$, $V$ and $\theta$ are shown, respectively, in Fig. $9 a$, b. For the case of $v_{w}=+1$, the magnitude of velocity components $(V, U)$ in the central region of the cavity is almost zero, which implies an almost stagnant fluid zone. This is evident from the contours of streamlines as given in Fig. 3. However, when the partial translating motion is applied about the left-top corner of the cavity, it causes substantial change next to the sidewalls in both the velocity profiles. The figures show that the velocity profiles reach to a peak value near the sidewalls, and a higher value is attained at $\mathrm{Ri}=100$. The reason for such behavior is understood by observing the streamlines in Fig. 3. With the increase in Ri value, as the buoyancy-induced fluid circulation becomes stronger than the shear-induced fluid circulation, the peak value of both the velocity components changes with respect to ' 0 ' line. In the case of $v_{w}=-1$, the buoyancy-induced fluid circulation governs both the velocity profiles indicating CCW flow direction. As the hot plumes (due to corner heating) rise upward along the right side wall and cold fluid goes downward along the left wall, the temperature profile of $\theta$ shows increasing trend along the mid-horizontal plane from the left wall to the top right wall. This temperature profile is severely affected by the direction of the partial translation of the walls. The temperature gradients at the vertical sidewalls (at $X=0,1$ ) represent local Nusselt number. Based on the direction of the partial sliding, the magnitude of temperature varies along the middle of the cavity. At lower value of $\mathrm{Ri}=0.1, \theta$ varies linearly, whereas at $\mathrm{Ri}=100$, the trend is almost varying sinusoidally. The variations in $V, U$ and $\theta$ are clearly reflected along with the variation of Re as presented in Fig. 9b. It is noted that, at a constant $\mathrm{Ri}$, the increase in Reynolds number has less impact on the mid-plane variables (compared to increase in Ri at fixed Re as in the top panel).

The influence of Darcy number on $U, V$ and $\theta$ is presented in Fig. $9 c$ (in the third row of Fig. 9). The figures show that the velocity magnitude close to the cavity wall is more for larger value of $\mathrm{Da}=10^{-3}$. The $U$-velocity profiles clearly demonstrate that the CCW flow velocity increases with the increase in Da value. This is true for both the velocity directions $\left(v_{w}= \pm 1\right)$, which can also be understood from the contour plots in Fig. 5. In Fig. 9c, the peak velocity of $U$ near the top wall is higher for the larger value of $\mathrm{Da}=10^{-3}$, whereas it becomes less in magnitude near the bottom wall. This happens so due to decreased fluid-flow resistance with the increase in $\mathrm{Da}$. Corresponding distribution of the mid-plane temperature (at $Y=0.5$ ) appears different at the sidewalls at $X=0$ and 1 . It is noted that the temperature $\theta$ varies nonlinearly inside the cavity. The partial sliding with $v_{w}=+1$, in contrast to that with $v_{w}=-1$, elevates temperature about the middle of the cavity. For both flow velocities $\left(v_{w}= \pm 1\right)$, at $\mathrm{Da}=10^{-7}$, the temperature variation almost linearly increases with $X$.

The influence of Hartmann number $\mathrm{Ha}$ is presented in Fig. 9d. The horizontal component of velocity in the whole cavity is small and almost constant, but it approaches to a positive value near the top wall due to the partial sliding imposed on this zone. The same effect is observed for the case of $v_{w}=-1$ where the $U$-component of velocity is approaching the maximum negative value near the same the top wall. The vertical component of velocity $V$ is maximized either positive or negative magnitude near the left sidewall. The rest of the zone of the cavity (up to the right sidewall), $V$, remains almost the same for all the values of $\mathrm{Ha}$ in either cases $\left(v_{w}= \pm 1\right)$. An almost similar patterns of variations in respective variables are noted with the variation of porosity $(\varepsilon)$ in Fig. 9e.

\section{Conclusions}

The study explores magnetohydrodynamic mixed convection in a porous enclosure with partial wall motion. The arrangements of corner heating and corner cooling are applied by dividing the bounding walls into two equal parts. The translational motion is applied on the cooler. The impact of partial translational motion is investigated considering a number of system parameters like Richardson number, Hartmann number, Reynolds number, Darcy number and porosity. The important findings from the study are listed below.

1. Importance and fundamental flow physics of a novel idea of partially driven cavity (PDC) are presented under the influence of magnetic force. Both assisting and opposing flow situations are investigated. Heat energy transport phenomena through porous media are presented with the Bejan's heatlines.

2. The thermal performance is well augmented through convection mode when the Grashof number increases. The Grashof number at higher value causes stronger fluid circulation in the cavity leading to increased heat transfer.

3. Heat transfer rate is found to increase substantially at enhanced Darcy number particularly at higher Richardson number. Similar trends of increasing heat transfer are observed with the increase in Reynolds number. Therefore, it is clear that the partial wall motion about a corner is a very effective mean for heat transfer. Heat transfer rate increases significantly at $\mathrm{Da}>10^{-5}$ for any directions of partial translation.

4. The increment of average Nu is much higher at $\mathrm{Ri}>10$. However, the direction of partial translation plays an important role on the thermal behavior. The velocity, 
$v_{w}=-1$, is always a better choice for higher heat transfer rate.

5. It is observed that Nu value is almost independent with the increase in porosity up to $\mathrm{Ri} \leq 10$, irrespective of the direction of partial translation. However, at $\mathrm{Ri}=100$, the average Nu increases significantly as the porosity increases.

6. The increase in magnetic effect (in terms of Hartmann number) dampens the buoyancy flow, leading to decreased heat transfer of the cavity. As Ha increases (keeping other parameter fixed) the average Nusselt number decreases; this decrement is higher at higher value of $\mathrm{Ri}=100$.

All these observations can provide useful information for the control of heat transport process in a thermal cavity and for future work using partially driven cavity.

Funding There is no financial support for this work.

\section{Compliance with ethical standards}

Conflict of interest The authors declare that they have no conflict of interest.

\section{References}

1. Sheikholeslami M (2017) Magnetohydrodynamic nanofluid forced convection in a porous lid driven cubic cavity using Lattice Boltzmann method. J Mol Liq 231:555-565

2. Biswas N, Manna NK (2018) Magneto-hydrodynamic Marangoni flow in bottom-heated lid-driven cavity. J Mol Liq 251:249-266

3. Rashidi S, Esfahani JA, Maskaniyan M (2017) Applications of Magnetohydrodynamics in biological systems - a review on the numerical studies. J Magn Magn Mater 439:358-372

4. Oztop HF, Salem KA, Pop I (2011) MHD mixed convection in a lid-driven cavity with corner heater. Int J Heat Mass Tranf 54:3494-3504

5. Ray S, Chatterjee D (2014) MHD mixed convection in a lid-driven cavity including heat conducting circular solid object and corner heaters with Joule heating. Int Commun Heat Mass Transf 57:200-207

6. Abu-Nada E (2015) Dissipative particle dynamics simulation of combined convection in a vertical lid driven cavity with a corner heater. Int J Thermal Sci. 92:72-84

7. Ismael MA, Abu-Nada E, Chamkha AJ (2017) Mixed convection in a square cavity filled with $\mathrm{CuO}$-water nanofluid heated by corner heater. Int J Mech Sci 133:42-50

8. Malleswaran A, Sivasankaran S (2016) A numerical simulation on MHD mixed convection in a lid-driven cavity with corner heaters. J Appl Fluid Mech 9:311-319

9. Begum AS, Nithyadevi N, Oztop HF, Al-Salem K (2017) Numerical simulation of MHD mixed convection in a nanofluid filled nonDarcy porous enclosure. Int J Mech Sci. 130:154-166

10. Ben Nasr K, Chouikh R, Kerkeni C, Guizani A (2006) Numerical study of the natural convection in cavity heated from the lower corner and cooled from the ceiling. Appl Therm Eng 26:772-775
11. Varol Y, Oztop HF, Koca A, Ozgen F (2009) Natural convection and fluid flow in inclined enclosure with a corner heater. Appl Therm Eng 29:340-350

12. Ahmed SE, Oztop HF, Salem KA (2014) Natural convection coupled with radiation heat transfer in an inclined porous cavity with corner heater. Comput Fluids 102:74-84

13. Sheremet MA, Oztop HF, Pop I, Salem KA (2016) MHD free convection in a wavy open porous tall cavity filled with nanofluids under an effect of corner heater. Int J Heat Mass Tranf 103:955-964

14. Hussain S, Mehmood K, Sagheer M (2016) MHD mixed convection and entropy generation of water-Alumina nanofluid flow in a double lid driven cavity with discrete heating. J Magn Magn Mater 419:140-155

15. Gibanov NS, Sheremet MA, Oztop HF, Abu-Hamdeh N (2017) Effect of uniform inclined magnetic field on mixed convection in a lid-driven cavity having a horizontal porous layer saturated with a ferrofluid. Int J Heat Mass Tranf 114:1086-1097

16. Chamkha AJ, Rashad AM, Armaghani T, Mansour MA (2018) Effects of partial slip on entropy generation and MHD combined convection in a lid-driven porous enclosure saturated with a Cu-water nanofluid. J Therm Anal Calorim 132:1291-1306

17. Bondarenko DS, Sheremet MA, Oztop HF, Abu-Hamdeh N (2019) Mixed convection heat transfer of a nanofluid in a lid-driven enclosure with two adherent porous blocks. JTherm Anal Calorim 135:1095-1105

18. Maghsoudi P, Siavashi M (2019) Application of nanofluid and optimization of pore size arrangement of heterogeneous porous media to enhance mixed convection inside a two-sided lid-driven cavity. J Therm Anal Calorim 135:947-961

19. Akar S, Rashidi S, Esfahani JA (2018) Second law of thermodynamic analysis for nanofluid turbulent flow around a rotating cylinder. J Therm Anal Calorim 132:1189-1200

20. Alsabery Al, Ismael MA, Chamkha AJ, Hashim I (2019) Effects of two-phase nanofluid model on MHD mixed convection in a lid-driven cavity in the presence of conductive inner block and corner heater. J Therm Anal Calorim 135:729-750

21. Sheikholeslami M, Chamkha AJ (2016) Flow and convective heat transfer of a ferro-nanofluid in a double-sided lid-driven cavity with a wavy wall in the presence of a variable magnetic field. Numer Heat Transf A 69:1186-1200

22. Selimefendigil F, Öztop Hakan F, Chamkha AJ (2016) MHD mixed convection and entropy generation of nanofluid filled lid driven cavity under the influence of inclined magnetic fields imposed to its upper and lower diagonal triangular domains. J Magn Magn Mater 406:266-281

23. Mohan CG, Satheesh A (2016) The numerical simulation of double-diffusive mixed convection flow in a lid-driven porous cavity with magnetohydrodynamic effect. Arab J Sci Eng 41:1867-1882

24. Chamkha AJ, Rashad AM, Mansour MA, Armaghani T, Ghalambaz $M$ (2017) Effects of heat sink and source and entropy generation on MHD mixed convection of a $\mathrm{Cu}$-water nanofluid in a lid-driven square porous enclosure with partial slip. Phys Fluids 29:052001-1-052001-22

25. Biswas N, Manna NK (2017) Enhanced convective heat transfer in lid-driven porous cavity with aspiration. Int J Heat Mass Tranf $114: 430-452$

26. Kimura S, Bejan A (1983) The heatline visualization of convective heat transfer. J Heat Transf 105:916-919

27. Biswas N, Mahapatra PS, Manna NK (2016) Enhanced thermal energy transport using adiabatic block inside lid driven cavity. Int J Heat Mass Tranf 100:407-427

28. Patankar SV (1980) Numerical heat transfer and fluid flow. Hemisphere, New York

29. Datta P, Mahapatra PS, Ghosh K, Manna NK, Sen S (2016) Heat transfer and entropy generation in a porous square 
enclosure in presence of an adiabatic block. Transp Porous Media 111:305-329

30. Ghasemi B, Aminossadati SM, Raisi A (2011) Magnetic field effect on natural convection in a nanofluid-filled square enclosure. Int J Therm Sci 50:1748-1756
Publisher's Note Springer Nature remains neutral with regard to jurisdictional claims in published maps and institutional affiliations. 